\title{
GLOBAL ASYMPTOTIC STABILIZATION OF THE SPINNING TOP
}

\author{
CHIH-JIAN WAN, VINCENT T. COPPOLA AND DENNIS S. BERNSTEIN \\ Department of Aerospace Engineering, The University of Michigan, Ann Arbor, MI 48109-2118, U.S.A.
}

\begin{abstract}
SUMMARY
We consider the problem of controlling a top to the sleeping motion using two different actuation schemes. For a fixed-base top two actuators are assumed to provide forces at the centre of mass in inertially fixed directions, while for a cart-mounted top two actuators are assumed to apply forces to the cart in inertially fixed directions. The controller for the cart-mounted top is obtained from the controller designed for the fixed-base top using d'Alembert's principle. Both controllers are proved to be globally asymptotically stabilizing. For the uncontrolled fixed-base top, necessary and sufficient conditions for Lyapunov stability of the sleeping motion are derived. For the case in which there is only one force actuator, locally asymptotically stabilizing control laws that drive the fixed-base top to the sleeping motion are also obtained.
\end{abstract}

KEY WORDS spinning top; global stabilization; Hamilton-Jacobi-Bellman theory; Lyapunov function; feedback linearization

\section{INTRODUCTION}

The spinning top is a widely studied problem in classical dynamics. Generally speaking, the spinning top is a special case of the motion of a heavy rigid body rotating under gravity with a fixed point. For a historical review and treatment of the general motion of the spinning top see References 1-7. In this paper we focus on Lagrange's top whose centre of mass lies on the axis of symmetry. ${ }^{8}$

Stability analysis of the sleeping motion of an uncontrolled Lagrange top mounted on a fixed base, i.e. a Lagrange top spinning with constant angular velocity and having its symmetric axis remain in the upright direction, is well developed. In References 9-12, Lyapunov's direct method was used, while in Reference 13 the authors applied the energy-Casimir method and in Reference 14 the authors applied the reduced Energy-Momentum method. Another approach to the stability of the sleeping motion is to investigate the behaviour of the roots of a cubic equation. ${ }^{15,16}$ Several attempts have also been made to analyse the stability of the sleeping motion by using linearized equations. ${ }^{17,18}$

In this paper we first investigate the Lyapunov stability of the sleeping motion of a fixed-base top by utilizing an alternative set of dynamical equations in terms of 2-1-3 Euler angles. Using this set of equations, we construct a Lyapunov function and derive necessary and sufficient conditions for Lyapunov stability of the sleeping motion. Related conclusions were first obtained by $\mathrm{Ge}$ and $\mathrm{Wu}^{10}$ using a quartic Lyapunov function.

CCC 0143-2087/95/030189-27

(C) 1995 by John Wiley \& Sons, Ltd.

Received 13 December

Revised 11 August 1994 
Next we consider the problem of controlling a top to the sleeping motion using two different actuation schemes. First we consider the case in which the vertex of the top is fixed in inertial space. Two actuators are assumed to provide forces at the centre of mass in inertially fixed directions. Because such actuators are difficult to realize in practice, we consider a second problem, that of a top spinning upon a cart which rolls upon the horizontal plane. The actuators for this problem apply forces to the cart in inertially fixed directions. We shall obtain the controller for the cart-mounted top from the controller designed for the fixed-base top by using d'Alembert's principle.

For the control problem involving a fixed-base top we consider the cases in which there are either one or two force inputs applied to the centre of mass of the top. For the case in which there are two force actuators we apply two design strategies, namely feedback linearization ${ }^{19-22}$ and the Hamilton-Jacobi-Bellman theory with zero dynamics, ${ }^{23}$ to obtain control laws that globally asymptotically stabilize the spinning top to the sleeping motion. It is shown that the control laws derived from the Hamilton-Jacobi-Bellman theory with zero dynamics asymptotically stabilize the spinning top to the sleeping motion globally (i.e. up to $90^{\circ}$ of tilt angle) even when the top is spinning arbitrarily slowly so that the sleeping motion of the uncontrolled top is unstable. For the case of only one force actuator we apply the Jurdjevic-Quinn technique to obtain control laws that locally asymptotically stabilize the spinning top to the sleeping motion. Performance functionals that are minimized by the corresponding control laws are also obtained.

\section{DERIVATION OF THE DYNAMICAL EQUATIONS FOR A SPINNING TOP}

In classical dynamics two methods have traditionally been used to describe the dynamics of a fixed-base top. The first is a set of six first-order differential equations involving the angular velocity vector and a unit vector in the negative gravity direction expressed in body coordinates, namely

$$
\begin{gathered}
\mathrm{J} \dot{\omega}=(-\boldsymbol{\omega} \times) \mathrm{J} \omega+m g \boldsymbol{\gamma} \times \mathbf{l} \\
\dot{\boldsymbol{\gamma}}=\boldsymbol{\gamma} \times \boldsymbol{\omega}
\end{gathered}
$$

where $x$ is the cross-product operator, $J$ is the inertia matrix taken at the vertex, $\omega=\left(\omega_{1}, \omega_{2}, \omega_{3}\right)^{\mathrm{T}}$ is the angular velocity of the top in body co-ordinates, $m$ is the mass of the top, $g$ is the local acceleration of gravity, 1 is the vector from the vertex to the centre of mass of the top expressed in body co-ordinates and $\boldsymbol{\gamma}=\left(\gamma_{1}, \gamma_{2}, \gamma_{3}\right)^{\mathrm{T}}$ is the unit vector in the negative gravity direction expressed in body co-ordinates. Equations (1) and (2), which are called the Euler-Poisson equations ${ }^{11}$, have been extensively studied during the $1950 \mathrm{~s}^{9,11,12}$ as well as in recent research. ${ }^{10,13}$

An alternative technique is to use Euler angles. Of the 12 possible sequences describing rigid body rotation, ${ }^{24,25}$ at least three have been used for the spinning top problem, specifically $3-1-3$ Euler angles, ${ }^{8} 3-2-3$ Euler angles ${ }^{17}$ and 3-2-1 Euler angles. ${ }^{15}$ The advantage of using these Euler angles is that the corresponding dynamical equations reduce to a single differential equation of the form

$$
\dot{\xi}^{2}=\hat{f}(\xi)=c\left(\xi-\xi_{1}\right)\left(\xi-\xi_{2}\right)\left(\xi-\xi_{3}\right)
$$

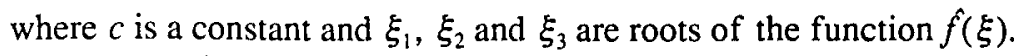

However, both methods mentioned above suffer serious drawbacks when there are external forces applied to the top. In the Euler-Poisson equations (1) and (2) the state variables do not specify the azimuth of the top axis, so the moment induced from external forces in fixed inertial directions cannot be expressed in terms of those state variables. In the $3-1-3,3-2-3$ and 
3-2-1 Euler angle formulations ${ }^{8.15 .26}$ as well as the 3-1-2 Euler angle formulation the sleeping motion does not correspond to an isolated equilibrium, since the first and third Euler angles are not distinguishable. These difficulties motivate us to develop an alternative set of dynamical equations for the controlled top.

A remedy for the above problem is to derive dynamical equations for the spinning top using Euler angles with an alternative sequence of rotations starting from the $X_{1}$ - or $X_{2}$-axis instead of the $X_{3}$ axis, where $\left(X_{1}, X_{2}, X_{3}\right)$ is the inertial reference frame and $X_{3}$ is in the negative gravity direction. There are eight possible sequences of rotations starting from the $X_{1}$ - or $X_{2}$-axis. However, only two of these eight sequences have dynamical equations with the origin as the sleeping motion, namely 2-1-3 and 1-2-3 Euler angles. For the remaining six sequences one can rewrite the dynamical equations in a translated co-ordinate system with the origin as the sleeping motion. Hence all eight of these sequences of rotations can be used to formulate the controlled top problem when there are external forces applied to the top. In this paper we use the 2-1-3 Euler angle formulation.

Let $\psi, \theta$ and $\phi$ denote 2-1-3 Euler angles from the inertial reference frame $\left(\mathrm{X}_{1}, X_{2}, X_{3}\right)$ to the body frame $\left(x_{1}, x_{2}, x_{3}\right)$, both of which have origin at the fixed vertex of the top (see Figure 1). If the two frames are initially coincident, the three Euler angles are defined by a sequence of rotations. First, a positive rotation of the $\left(X_{1}, X_{2}, X_{3}\right)$ frame by an angle $\psi$ about the $X_{2}$-axis results in the frame $\left(x_{1}^{\prime}, x_{2}^{\prime}, x_{3}^{\prime}\right)$. Next, a positive rotation of the $\left(x_{1}^{\prime}, x_{2}^{\prime}, x_{3}^{\prime}\right)$ frame by an angle $\theta$ about the $x_{1}^{\prime}$-axis results in the $\mathscr{F}$-frame $\left(x_{1}^{\prime \prime}, x_{2}^{\prime \prime}, x_{3}^{\prime \prime}\right)$. Finally, a positive rotation of the $F$-frame by an angle $\phi$ about the $x_{3}^{\prime \prime}$-axis results in the body frame $\left(x_{1}, x_{2}, x_{3}\right)$, which is attached to the top. Note that the F-frame precesses but does not spin with the top. Hence the angular velocity $\omega$ of the top expressed in the $\mathscr{F}$-frame is

$$
\boldsymbol{\omega}=\left[\begin{array}{l}
0 \\
0 \\
\dot{\phi}
\end{array}\right]+\omega_{\mathscr{F}}=\left[\begin{array}{l}
\dot{\theta} \\
\dot{\psi} \cos \theta \\
\dot{\phi}-\dot{\psi} \sin \theta
\end{array}\right]
$$

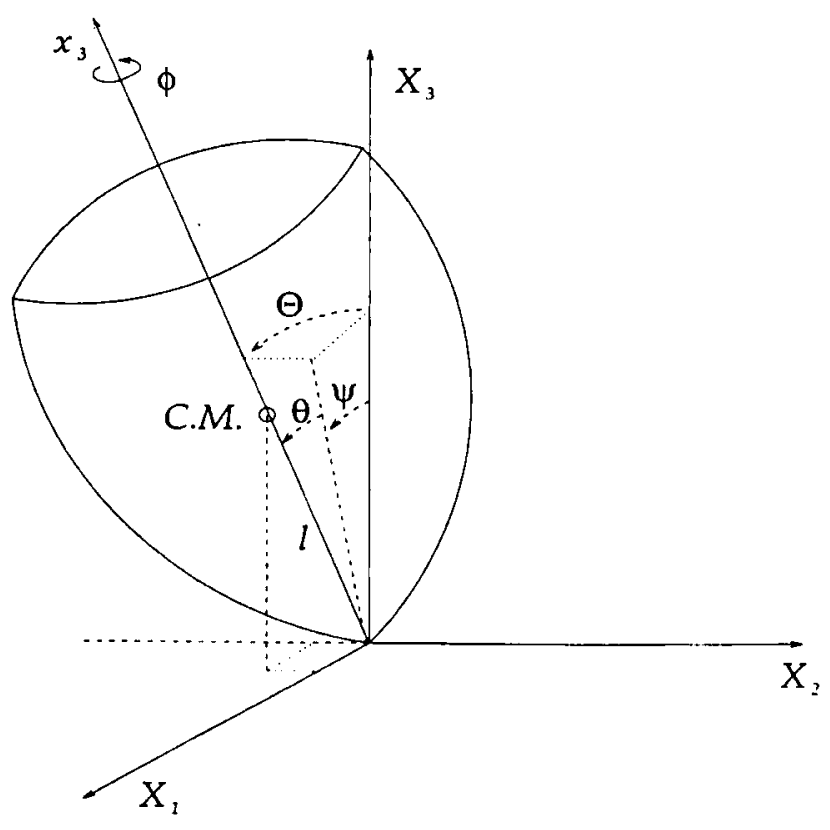

Figure 1. Definition of 2-1-3 Euler angles 
where $\omega_{\mathscr{F}}=(\dot{\theta}, \dot{\psi} \cos \theta, \dot{\psi} \sin \theta)^{\mathrm{T}}$ is the angular velocity of the $\mathscr{F}$-frame with respect to the inertial reference frame.

For a fixed-base top the kinetic and potential energy are given by

$$
\begin{gathered}
T=\frac{1}{2} J_{1}\left(\dot{\theta}^{2}+\dot{\psi}^{2} \cos ^{2} \theta\right)+\frac{1}{2} J_{3}(\dot{\phi}-\dot{\psi} \sin \theta)^{2} \\
V_{\mathrm{P}}=m g l \cos \theta \cos \psi
\end{gathered}
$$

where $\mathbf{J}=\operatorname{diag}\left(J_{1}, J_{1}, J_{3}\right)$ is the principal inertia matrix of the top with respect to the vertex and $l$ is the distance between the vertex and the centre of mass. Note that we have assumed a symmetric top. The Lagrangian $\mathscr{L}$ is then

$$
\mathscr{L}=T-V_{\mathrm{P}}=\frac{1}{2} J_{1}\left(\dot{\theta}^{2}+\dot{\psi}^{2} \cos ^{2} \theta\right)+\frac{1}{2} J_{3}(\dot{\phi}-\dot{\psi} \sin \theta)^{2}-m g l \cos \theta \cos \psi
$$

The generalized momentum corresponding to $\phi$ is

$$
p_{\phi} \triangleq \frac{\partial \mathscr{L}}{\partial \dot{\phi}}=J_{3}(\dot{\phi}-\dot{\psi} \sin \theta)=J_{3} \Omega
$$

where the total spin $\Omega$ is defined as

$$
\Omega \triangleq \dot{\phi}-\dot{\psi} \sin \theta
$$

Since $\phi$ does not appear in the Lagrangian $\mathscr{L}$, it is an ignorable co-ordinate. If the generalized force corresponding to $\phi$ is zero, then $p_{\phi}=J_{3} \Omega$ is constant, hence $\Omega$ is constant. By defining the Routhian $R_{o}$ as

$$
R_{o} \triangleq \mathscr{L}-p_{\phi} \dot{\phi}=\frac{1}{2} J_{1}\left(\dot{\theta}^{2}+\dot{\psi}^{2} \cos ^{2} \theta\right)-\frac{1}{2 J_{3}} p_{\phi}^{2}-p_{\phi} \dot{\psi} \sin \theta-m g l \cos \theta \cos \psi
$$

Lagrange's equation becomes

$$
\frac{\mathrm{d}}{\mathrm{d} t}\left(\frac{\partial R_{o}}{\partial \dot{q}}\right)-\frac{\partial R_{o}}{\partial q}=Q
$$

where $q$ and $Q$ are the generalized co-ordinate and generalized force respectively. ${ }^{27}$ For uncontrolled tops the generalized force $Q$ is zero. In this case, substituting equation (10) into (11) yields

$$
\begin{gathered}
J_{1} \ddot{\theta}+J_{1} \dot{\psi}^{2} \cos \theta \sin \theta+p_{\phi} \dot{\psi} \cos \theta-m g l \sin \theta \cos \psi=0 \\
J_{1} \ddot{\psi} \cos ^{2} \theta-2 J_{1} \dot{\psi} \dot{\theta} \cos \theta \sin \theta-p_{\phi} \dot{\theta} \cos \theta-m g l \cos \theta \sin \psi=0
\end{gathered}
$$

The tilt angle $\Theta$, the angle between the $X_{3}$-and $x_{3}$-axis, is defined by

$$
\Theta=\cos ^{-1}(\cos \theta \cos \psi)
$$

If the tilt angle $\Theta$ is constant for all $t>t_{1}$, then the top is in steady precession. If steady precession occurs, then

$$
\begin{aligned}
& \dot{\theta} \sin \theta \cos \psi+\dot{\psi} \cos \theta \sin \psi=0 \\
& \dot{\psi}=\frac{\sin \theta}{2 J_{1} \cos ^{2} \theta}\left[p_{\phi} \pm \sqrt{ }\left(p_{\phi}^{2}-4 J_{1} m g l \cos \theta \cos \psi\right)\right] \\
& p_{\phi}^{2}>4 J_{1} m g l \cos \theta \cos \psi
\end{aligned}
$$


Conditions (15)-(17) give necessary conditions for the steady precession of a fixed-base top in terms of 2-1-3 Euler angles. Note that condition (17) agrees with the usual 3-2-1 Euler angle description for steady precession. ${ }^{15}$. Also note that in using the Euler-Poisson equations (1) and (2), the tilt angle $\Theta$ is defined by $\cos ^{-1} \gamma_{3}$.

To obtain first-order equations, let $x_{1}=\theta, x_{2}=\dot{\theta}, x_{3}=\psi$ and $x_{4}=\dot{\psi}$, so that equations (12) and (13) become

$$
\dot{\mathbf{x}}=\mathbf{f}(\mathbf{x}) \triangleq\left[\begin{array}{c}
x_{2} \\
-\left(x_{4}^{2} / 2\right) \sin \left(2 x_{1}\right)-b x_{4} \cos x_{1}+(c / 2) \sin x_{1} \cos x_{3} \\
x_{4} \\
\left(2 x_{2} x_{4} \sin x_{1}+b x_{2}+(c / 2) \sin x_{3}\right) / \cos x_{1}
\end{array}\right]
$$

where $\mathbf{x} \triangleq\left(x_{1}, x_{2}, x_{3}, x_{4}\right)^{\mathrm{T}}$ and $b$ and $c$ are defined following Greenwood, ${ }^{15}$ namely

$$
b \triangleq p_{\phi} / J_{1}, \quad c \triangleq 2 m g l / J_{1}
$$

In the following we consider the domain $\mathscr{D}$ defined by

$$
\mathscr{D} \triangleq\left\{\mathrm{x} \in \mathbb{R}^{4} \mid x_{1}, x_{3} \in(-\pi / 2, \pi / 2), x_{2}, x_{4} \in \mathbb{R}\right\}
$$

which corresponds to top motion with tilt angle $\Theta$ less than $90^{\circ}$. We shall examine the stability of (18) in the domain $\mathscr{D}$. Note that equation (18) has an isolated equilibrium at the origin which corresponds to the sleeping motion of the top. Linearizing equation (18) with respect to the origin yields

$$
\dot{\mathbf{x}}=\mathbf{A x} \triangleq\left[\begin{array}{cccc}
0 & 1 & 0 & 0 \\
c / 2 & 0 & 0 & -b \\
0 & 0 & 0 & 1 \\
0 & b & c / 2 & 0
\end{array}\right] \mathbf{x}
$$

The corresponding second-order equation is

$$
\left[\begin{array}{l}
\ddot{x}_{1} \\
\ddot{x}_{3}
\end{array}\right]+\left[\begin{array}{rr}
0 & b \\
-b & 0
\end{array}\right]\left[\begin{array}{l}
\dot{x}_{1} \\
\dot{x_{3}}
\end{array}\right]+\left[\begin{array}{cc}
-c / 2 & 0 \\
0 & -c / 2
\end{array}\right]\left[\begin{array}{l}
x_{1} \\
x_{3}
\end{array}\right]=\left[\begin{array}{l}
0 \\
0
\end{array}\right]
$$

which is equivalent to the result obtained by Bahar $^{17}$ using 3-2-3 Euler angles. In that derivation the vertical motion of the centre of mass was neglected in formulating the Routhian, so that the complete non-linear equations of motion were not given. One goal of the present paper has been to derive the complete non-linear equations for the spinning top. In the next section we investigate the stability of the sleeping motion of a fixed-base top. In Section 7 we will derive controlled dynamical equations for the spinning top on a moving cart using the same procedures as in this section.

\section{LYAPUNOV STABILITY ANALYSIS OF THE SLEEPING MOTION}

Lyapunov stability of the sleeping motion of a fixed-base top has been studied by many earlier researchers. ${ }^{9-16}$ In References $9-12$ the authors used the Euler-Poisson equations (1) and (2) and constructed a Lyapunov function based on perturbations of (1) and (2). In Reference 13 the the energy-Casimir method was applied to construct a Lyapunov function based on the same set of equations. Also, in Reference 14 the authors applied the reduced Energy-Momentum method to demonstrate nonlinear orbital stability of the 
sleeping motion. Another classical approach to the stability of the sleeping motion is to investigate the behaviour of the roots of the cubic equation (3). ${ }^{15.16}$ In this section we analyse the Lyapunov stability of the sleeping motion using (18) based on the 2-1-3 Euler angle formulation.

\subsection{Stability of the linearized equation}

For the linearized equation (21), if $b^{2}>2 c$, then the eigenvalues of $\mathbf{A}$ are $\pm \mathrm{j} \omega_{1}$ and $\pm \mathrm{j} \omega_{2}$, where $\mathrm{j}=\sqrt{ }(-1)$ and

$$
\begin{aligned}
& \omega_{1} \triangleq \sqrt{\left(\frac{1}{2}\left(b^{2}-c\right)-\frac{b}{2} \sqrt{ }\left(b^{2}-c\right)\right)} \\
& \omega_{2} \triangleq \sqrt{\left(\frac{1}{2}\left(b^{2}-c\right)+\frac{b}{2} \sqrt{ }\left(b^{2}-2 c\right)\right)}
\end{aligned}
$$

If $b^{2}>2 c$, then all four eigenvalues are distinct, while if $b^{2}=2 c$, then these four eigenvalues are repeated complex conjugate pairs. If $b^{2}<2 c$, then the eigenvalues of $\mathbf{A}$ are

$$
\pm j\left(\frac{1}{2}\left(b^{2}-c\right) \pm j \frac{b}{2} \sqrt{ }\left(2 c-b^{2}\right)\right)^{1 / 2}
$$

so that $\mathbf{A}$ has two eigenvalues in the open right half-plane, which corresponds to instability of the linearized equation (21) and thus instability of the non-linear equation (18). We consider the case $b^{2}>2 c$ here and we will return to the case $b^{2}=2 c$ in Section 3.2.

Suppose that $b^{2}>2 c$ so that the zero solution of $\dot{\mathbf{x}}=\mathbf{A x}$ is Lyapunov-stable with four distinct eigenvalues of $\mathbf{A}$ on the imaginary axis. In this case $\mathbf{A}$ is similar to its real Jordan form $\mathbf{A}_{\mathrm{N}}$ through an invertible matrix $\mathbf{S} \in \mathbb{R}^{4 \times 4}$, namely ${ }^{28}$

$$
\mathbf{A}=\mathbf{S A}_{\mathrm{N}} \mathbf{S}^{-1}
$$

The eigenvectors corresponding to the distinct eigenvalues $\mathrm{j} \omega_{1}$ and $\mathrm{j} \omega_{2}$ are

$$
\left[\begin{array}{c}
1+j \\
-\omega_{1}(1-j) \\
\frac{b-\sqrt{ }\left(b^{2}-2 c\right)}{2 \omega_{1}}(1-j) \\
\frac{b-\sqrt{(}\left(b^{2}-2 c\right)}{2}(1+j)
\end{array}\right], \quad\left[\begin{array}{c}
1+j \\
-\omega_{2}(1-j) \\
\frac{b+\sqrt{ }\left(b^{2}-2 c\right)}{2 \omega_{2}}(1-j) \\
\frac{b+\sqrt{ }\left(b^{2}-2 c\right)}{2}(1+j)
\end{array}\right]
$$

Taking the real and imaginary parts of the above eigenvectors yields

$$
\mathbf{S}=\left[\begin{array}{cccc}
1 & 1 & 1 & 1 \\
-\omega_{2} & \omega_{2} & -\omega_{1} & \omega_{1} \\
\frac{b+\sqrt{ }\left(b^{2}-2 c\right)}{2 \omega_{2}} & -\frac{b+\sqrt{ }\left(b^{2}-2 c\right)}{2 \omega_{2}} & \frac{b-\sqrt{ }\left(b^{2}-2 c\right)}{2 \omega_{1}} & -\frac{b-\sqrt{ }\left(b^{2}-2 c\right)}{2 \omega_{1}} \\
\frac{b+\sqrt{ }\left(b^{2}-2 c\right)}{2} & \frac{b+\sqrt{ }\left(b^{2}-2 c\right)}{2} & \frac{b-\sqrt{ }\left(b^{2}-2 c\right)}{2} & \frac{b-\sqrt{ }\left(b^{2}-2 c\right)}{2}
\end{array}\right]
$$


while $\mathbf{A}_{\mathrm{N}}$ is given by

$$
\mathbf{A}_{\mathrm{N}}=\left[\begin{array}{cccc}
0 & \omega_{2} & 0 & 0 \\
-\omega_{2} & 0 & 0 & 0 \\
0 & 0 & 0 & \omega_{1} \\
0 & 0 & -\omega_{1} & 0
\end{array}\right]
$$

To show directly that $\mathbf{A}$ is Lyapunov-stable, let $V(\mathbf{x})=\mathbf{x}^{\mathbf{T}} \mathbf{P} \mathbf{x}$, where the symmetric matrix $\mathbf{P}$ is given by

$$
\mathbf{P}=\mathbf{S}^{-\mathbf{T}} \mathbf{S}^{-1}=\frac{1}{b^{2}-2 c}\left[\begin{array}{cccc}
\left(b^{2}-c\right) / 2 & 0 & 0 & -b / 2 \\
0 & 1 & b / 2 & 0 \\
0 & b / 2 & \left(b^{2}-c\right) / 2 & 0 \\
-b / 2 & 0 & 0 & 1
\end{array}\right]
$$

It is straightforward to check that $\mathbf{P}>0$ if and only if $b^{2}>2 c$. The time derivative of $V(\mathbf{x})$ is

$$
\dot{V}(\mathbf{x})=\mathbf{x}^{\mathrm{T}}\left(\mathbf{A}^{\mathrm{T}} \mathbf{P}+\mathbf{P A}\right) \mathbf{x}=\mathbf{x}^{\mathrm{T}} \mathbf{S}^{-\mathrm{T}}\left(\mathbf{A}_{\mathrm{N}}^{\mathrm{T}}+\mathbf{A}_{\mathrm{N}}\right) \mathbf{S}^{-1} \mathbf{x}=0
$$

Although Lyapunov stability of the linearized system does not guarantee Lyapunov stability of the non-linear system, the form of $\mathbf{P}$ will be useful for constructing a Lyapunov function for the non-linear equation. Note that attempts to show the stability of the sleeping motion using the linearized equation are inconclusive so far as Lyapunov stability is concerned. ${ }^{17,18}$

\subsection{Lyapunov stability of the non-linear equation}

Now we consider the Lyapunov stability of the non-linear equation (18). In References 9-12 Lyapunov functions are constructed to prove Lyapunov stability of the sleeping motion using the Euler-Poisson equations. These Lyapunov functions, which are based on Chetayev's construction, ${ }^{9}$ are quadratic. The energy-Casimir method ${ }^{13}$ gives a larger family of Lyapunov functions which are not necessarily quadratic but which have quadratic terms that are still required to be positive definite. Stability analysis using the $3-1-3,3-2-1$ or 3-2-3 Euler angle formulations was given in References 8,15, 16 and 18. In Reference 14 the authors used the reduced Energy-Momentum method to show that the sleeping motion is orbitally non-linearly stable. All these results require that $b^{2}>2 c$. For the case $b^{2}=2 c, \mathrm{Ge}$ and $\mathrm{Wu}^{10}$ used the Euler-Poisson equations and a quartic Lyapunov function to show Lyapunov stability of the sleeping motion and thus proved for the first time that the sleeping motion of a spinning top is Lyapunov-stable if and only if $b^{2} \geqslant 2 c$. Here we construct Lyapunov functions for (18) based on the Energy-Casimir method ${ }^{13.29 .30}$ which takes advantage of the conserved quantities embedded in (18).

For (18) there are two constants of motion, namely energy $h_{\mathrm{cl}}(\mathbf{x})$ and vertical angular momentum $h_{\mathrm{c} 2}(\mathbf{x})$ given by

$$
\begin{gathered}
h_{\mathrm{c} 1}(\mathbf{x}) \triangleq \frac{1}{2}\left(x_{2}^{2}+x_{4}^{2} \cos ^{2} x_{1}\right)+\frac{c}{2} \cos x_{1} \cos x_{3} \\
h_{\mathrm{c} 2}(\mathbf{x}) \triangleq-x_{2} \sin x_{3}+x_{4} \sin x_{1} \cos x_{1} \cos x_{3}+b \cos x_{1} \cos x_{3}
\end{gathered}
$$


By defining

$$
\begin{aligned}
V(\mathbf{x})= & h_{\mathrm{c} 1}(\mathbf{x})-h_{\mathrm{c} 1}(\mathbf{0})-\frac{b}{2}\left[h_{\mathrm{c} 2}(\mathbf{x})-h_{\mathrm{c} 2}(\mathbf{0})\right] \\
= & \frac{1}{2}\left(x_{2}^{2}+x_{4}^{2} \cos ^{2} x_{1}\right)+\frac{c}{2} \cos x_{1} \cos x_{3} \\
& -\frac{c}{2}-\frac{b}{2}\left(-x_{2} \sin x_{3}+x_{4} \sin x_{1} \cos x_{1} \cos x_{3}+b \cos x_{1} \cos x_{3}-b\right)
\end{aligned}
$$

it is easy to show that $V(\mathbf{0})=0$ and $V^{\prime}(\mathbf{0})=0$, where $V^{\prime}(\mathbf{x})$ is the gradient of $V(\mathbf{x})$. Furthermore, the Hessian $V^{\prime \prime}(\mathbf{x})$ of $V(\mathbf{x})$ evaluated at $\mathbf{0}$ is given by

$$
V^{\prime \prime}(\mathbf{0})=\left(b^{2}-2 c\right) \mathbf{P}
$$

where $\mathbf{P}$ is defined as in (29). Since, as shown in Section $3.1, b^{2}>2 c$ implies $\mathbf{P}>0$, it follows that $V(\mathbf{x})$ defined in (32) is (at least) locally positive definite. Furthermore, since $V(\mathbf{x})$ is a combination of constants of motion, its time derivative is identically zero, i.e. $\dot{V}(\mathbf{x})=0$. Consequently, if $b^{2}>2 c$, then $V(\mathbf{x})$ is a Lyapunov function that proves Lyapunov stability of the sleeping motion. Note that this development does not address the case $b^{2}=2 c$, which is considered below.

\section{Remark 3.1}

It should be noted that the Lyapunov function constructed in (32) is not the only choice for guaranteeing Lyapunov stability of the sleeping motion. For example,

$$
\begin{aligned}
V(\mathbf{x})= & h_{\mathrm{c} 1}(\mathbf{x})-h_{\mathrm{c} 1}(\mathbf{0})-\frac{1}{4 J_{1}}\left[h_{\mathrm{c} 2}(\mathbf{x})-h_{\mathrm{c} 2}(\mathbf{0})\right]^{2} \\
= & \frac{1}{2}\left(x_{2}^{2}+x_{4}^{2} \cos ^{2} x_{1}\right)+\frac{c}{2} \cos x_{1} \cos x_{3}-\frac{c}{2} \\
& -\frac{1}{4 J_{1}}\left(-x_{2} \sin x_{3}+x_{4} \sin x_{1} \cos x_{1} \cos x_{3}+b \cos x_{1} \cos x_{3}-b\right)^{2}
\end{aligned}
$$

will also suffice if $b^{2}>2 c$. It is easy to check that the Lyapunov function (34) also satisfies $V(\mathbf{0})=0, V^{\prime}(\mathbf{0})=\mathbf{0}$ and $(33)$.

Using the Lyapunov function (32), we now give necessary and sufficient conditions for Lyapunov stability of the sleeping motion for the case $b^{2}>2 c$. This result simplifies the result given by $\mathrm{Ge}$ and $\mathrm{Wu} .^{10}$

\section{Proposition 1}

The sleeping motion of a fixed-base top is Lyapunov-stable if and only if $b^{2}>2 c$.

Proof. In Section 3.1 it was shown that if $b^{2}<2 c$, then the sleeping motion is unstable. To show stability in the case $b^{2}>2 c$, we can rewrite the Lyapunov function (32) as

$$
\begin{aligned}
V(\mathbf{x})= & \frac{1}{2}\left(x_{2}+\frac{b}{2} \sin x_{3}\right)^{2}+\frac{1}{2}\left(x_{4} \cos x_{1}-\frac{b}{2} \sin x_{1} \cos x_{3}\right)^{2} \\
& +\frac{1}{4}\left(b^{2}-2 c\right)\left(1-\cos x_{1} \cos x_{3}\right)+\frac{b^{2}}{8}\left(1-\cos x_{1} \cos x_{3}\right)^{2}
\end{aligned}
$$


It can be seen from (35) that if $b^{2}>2 c$, then $V(\mathbf{x})>0, \mathbf{x} \in \mathscr{D}$. Furthermore, it can be shown that $V(\mathbf{x})=0$ implies that $\mathbf{x}=\mathbf{0}$. Hence, if $b^{2}>2 c, V(\mathbf{x})$ is positive definite for all $\mathbf{x} \in \mathscr{D}$. This completes the proof.

It should be noted that in the limiting case $b^{2}=2 c, V(\mathbf{x})$ is positive definite for all $\mathbf{x} \in \mathscr{D}$ even though its Hessian $V^{\prime \prime}(\mathbf{0})$ is non-negative definite. Thus it is not surprising that $\mathrm{Ge}$ and $\mathrm{Wu}^{10}$ required a quartic Lyapunov function to supplement the non-negative definite quadratic terms in the case $b^{2}=2 c$.

\section{DYNAMICAL EQUATIONS FOR THE CONTROLLED TOP}

In this section we derive equations for the controlled fixed-base top. We assume that two control forces $u_{1}$ and $u_{2}$ are applied to the centre of mass of the top along the inertial (horizontal) directions $X_{1}, X_{2}$. In Section 5 we will study the controlled top problem by using two control forces, whereas in Section 6 we consider the same problem with only one force actuator by eliminating $u_{1}$.

Suppose that two control forces $u_{1}$ and $u_{2}$ are applied to the centre of mass of the top along the inertial (horizontal) directions $X_{1}$ and $X_{2}$ directions, then the generalized force $Q$ has the components

$$
\mathbf{Q}=\left[\begin{array}{l}
Q_{\theta} \\
Q_{\psi}
\end{array}\right]=\left[\begin{array}{c}
-l\left(u_{1} \sin \theta \sin \psi+u_{2} \cos \theta\right) \\
l u_{1} \cos \theta \cos \psi
\end{array}\right]
$$

Substituting (36) into (11) yields the controlled dynamical equations

$$
\begin{gathered}
J_{1} \ddot{\theta}+J_{1} \dot{\psi}^{2} \cos \theta \sin \theta+p_{\phi} \dot{\psi} \cos \theta-m g l \sin \theta \cos \psi=-l\left(u_{1} \sin \theta \sin \psi+u_{2} \cos \theta\right) \\
J_{1} \ddot{\psi} \cos ^{2} \theta-2 J_{1} \dot{\psi} \dot{\theta} \cos \theta \sin \theta-p_{\phi} \dot{\theta} \cos \theta-m g l \cos \theta \sin \psi=l u_{1} \cos \theta \cos \psi
\end{gathered}
$$

In first-order form, (37) and (38) become

$$
\dot{\mathbf{x}}=\mathbf{f}(\mathbf{x})+\mathbf{g}(\mathbf{x}) \mathbf{u}
$$

where $\mathbf{f}(\mathbf{x})$ is defined in (18),

$$
\mathbf{g}(\mathbf{x}) \triangleq\left[g_{1}(\mathbf{x}), g_{2}(\mathbf{x})\right]=\frac{l}{J_{1}}\left[\begin{array}{cc}
0 & 0 \\
-\sin x_{1} \sin x_{3} & -\cos x_{1} \\
0 & 0 \\
\cos x_{3} / \cos x_{1} & 0
\end{array}\right]
$$

and $\mathbf{u}=\left(u_{1}, u_{2}\right)^{\top} \in \mathbb{R}^{2}$. It is straightforward to check that the linearization of (39) about the origin is controllable, so that linear control laws based upon the linearization of (39) locally asymptotically stabilize (39) to the origin. In the next section we synthesize non-linear control laws that globally asymptotically stabilize (39) on $\mathscr{D}$.

\section{FEEDBACK STABILIZATION WITH TWO FORCE ACTUATORS}

In this section we consider the case of using two force actuators to the centre of mass of a fixed-base top and apply two different control strategies, namely feedback linearization ${ }^{20-22}$ and the Hamilton-Jacobi-Bellman (HJB) theory with zero dynamics, ${ }^{23,31,32}$ to synthesize control laws that stabilize the spinning top to the sleeping motion. We briefly review each control technique to clarify notation and assumptions. Further details can be found in Reference 21 as 
well as in References 23, 31 and 32 and references therein. For generality we consider a nonlinear controlled system which is affine in the control of the form

$$
\dot{\mathbf{x}}=\mathbf{f}(\mathbf{x})+\mathbf{g}(\mathbf{x}) \mathbf{u}=\mathbf{f}(\mathbf{x})+\mathbf{g}_{1}(\mathbf{x}) \mathbf{u}_{1}+\cdots+\mathbf{g}_{m}(\mathbf{x}) \mathbf{u}_{m}
$$

where $\mathbf{x} \in \mathscr{X} \subset \mathbb{R}^{n}$, with $\mathscr{X}$ having the origin as an interior point, $\mathbf{u} \in \mathbb{R}^{m}$ and $\mathbf{g}_{1}(\mathbf{x}), \ldots, \mathbf{g}_{m}(\mathbf{x})$ are column vectors of $\mathbf{g}(\mathbf{x})$. We assume that $\mathbf{f}$ and $\mathbf{g}_{1}, \ldots, \mathbf{g}_{m}$ are sufficiently smooth and without loss of generality we assume that the origin is an equilibrium state, namely $\mathbf{f}(\mathbf{0})=\mathbf{0}$.

\subsection{Feedback linearization}

The non-linear controlled system (40) is feedback-linearizable $e^{20.22}$ if there exists a coordinate transformation that transforms the system to a companion form and a non-linear feedback control law that cancels the non-linear dynamics, thus reducing the original system to a linear controllable system.

We recall several definitions from reference 21 which are needed for our development. A $k$ dimensional vector field $\mathbf{f}_{1}(\mathbf{x}), \mathbf{f}_{2}(\mathbf{x}), \ldots, \mathbf{f}_{k}(\mathbf{x})$, defined on the open subset $\mathscr{D}_{0}$ of $\mathscr{X}$, is a mapping that assigns a $k$-dimensional vector to each point $\mathbf{x}$ of $\mathscr{D}_{0}$.

\section{Definition 1}

A $k$-dimensional distribution $\Delta(\cdot)$ on $\mathscr{D}_{0}$ is a map that assigns to each $\mathbf{x} \in \mathscr{D}_{0}$, a $k$-dimensional subspace $\Delta(\mathbf{x})$ of $\mathscr{X}$ such that there exists a $k$-dimensional vector field $\mathbf{f}_{1}(\mathbf{x}), \mathbf{f}_{2}(\mathbf{x}), \ldots, \mathbf{f}_{k}(\mathbf{x})$ satisfying

(i) $\left\{\mathbf{f}_{1}(\mathbf{x}), \mathbf{f}_{2}(\mathbf{x}), \ldots, \mathbf{f}_{k}(\mathbf{x})\right\}$ is a linearly independent set, $\mathbf{x} \in \mathscr{D}_{0}$

(ii) $\Delta(\mathbf{x})=\operatorname{span}\left\{\mathbf{f}_{1}(\mathbf{x}), \mathbf{f}_{2}(\mathbf{x}), \ldots, \mathbf{f}_{k}(\mathbf{x})\right\}, \mathbf{x} \in \mathscr{D}_{0}$.

We let $\left[\mathbf{f}_{i}, \mathbf{f}_{j}\right]$ denote the Lie bracket

$$
\left[\mathbf{f}_{i}, \mathbf{f}_{j}\right]=\operatorname{ad}_{f_{i}} \mathbf{f}_{j} \triangleq \frac{\partial \mathbf{f}_{j}}{\partial \mathbf{x}} \mathbf{f}_{i}-\frac{\partial \mathbf{f}_{i}}{\partial \mathbf{x}} \mathbf{f}_{j}
$$

and define higher-order and zero-order Lie brackets by

$$
\operatorname{ad}_{f_{i}}^{k} \mathbf{f}_{j}=\operatorname{ad}_{f_{i}} \operatorname{ad}_{f_{i}}^{k-1} \mathbf{f}_{j}, \quad \quad \operatorname{ad}_{f_{i}}^{0} \mathbf{f}_{j}=\mathbf{f}_{j}
$$

\section{Definition 2}

The distribution $\Delta(\mathbf{x})$ is involutive if $\left[\mathbf{f}_{i}, \mathbf{f}_{j}\right] \in \Delta(\mathbf{x}), \mathbf{f}_{i}, \mathbf{f}_{j} \in \Delta(\mathbf{x})$.

For the controlled system (40) define the distributions

$$
\begin{aligned}
& \Delta_{0}(\mathbf{x}) \triangleq \operatorname{span}\left\{\mathbf{g}_{1}(\mathbf{x}), \mathbf{g}_{2}(\mathbf{x}), \ldots, \mathbf{g}_{m}(\mathbf{x})\right\} \\
& \Delta_{1}(\mathbf{x}) \triangleq \operatorname{span}\left\{\mathbf{g}_{1}(\mathbf{x}), \mathbf{g}_{2}(\mathbf{x}), \ldots, \mathbf{g}_{m}(\mathbf{x}), \operatorname{ad}_{f} \mathbf{g}_{1}(\mathbf{x}), \operatorname{ad}_{f} \mathbf{g}_{2}(\mathbf{x}), \ldots, \operatorname{ad}_{f} \mathbf{g}_{m}(\mathbf{x})\right\} \\
& \Delta_{i}(\mathbf{x}) \triangleq \operatorname{span}\left\{\operatorname{ad}_{f}^{k} \mathbf{g}_{j}(\mathbf{x}), k=0,1, \ldots, i, j=1,2, \ldots, m\right\}
\end{aligned}
$$

The following theorem is due to Su et al. ${ }^{20}$ see also Reference 21. 


\section{Theorem 1}

Suppose rank $\mathbf{g}(0)=m$. Then (40) is feedback-linearizable if and only if

(i) for each $0<i<n-1$, the distribution $\Delta_{i}(\mathbf{x})$ has constant dimension in a neighbourhood of the origin

(ii) the distribution $\Delta_{n-1}(\mathbf{x})$ has constant dimension $n$ in a neighbourhood of the origin

(iii) for each $0<i<n-2$ the distribution $\Delta_{i}(\mathbf{x})$ is involutive in a neighbourhood of the origin.

Now consider the controlled top equation (39). It is easy to check that $\operatorname{dim} \Delta_{0}(\mathbf{x})=2$ and $\operatorname{dim} \Delta_{l}(x)=4$ for all $x \in \mathscr{D}$, where

$$
\begin{gathered}
\Delta_{0}(\mathbf{x})=\operatorname{span}\left\{\mathbf{g}_{1}(\mathbf{x}), \mathbf{g}_{2}(\mathbf{x})\right\}=\operatorname{span}\left(\frac{l}{J_{1}}\left[\begin{array}{c}
0 \\
-\sin x_{1} \sin x_{3} \\
0 \\
\cos x_{3} / \cos x_{1}
\end{array}\right], \frac{l}{J_{1}}\left[\begin{array}{c}
0 \\
-\cos x_{1} \\
0 \\
0
\end{array}\right]\right) \\
\Delta_{1}(\mathbf{x})=\operatorname{span}\left\{\mathbf{g}_{1}(\mathbf{x}), \mathbf{g}_{2}(\mathbf{x}), \operatorname{ad}_{f} \mathbf{g}_{1}(\mathbf{x}), \operatorname{ad}_{f} \mathbf{g}_{2}(\mathbf{x})\right\}
\end{gathered}
$$

with

$$
\begin{gathered}
\operatorname{ad}_{f} \mathbf{g}_{1}(\mathbf{x})=\frac{l}{J_{1}}\left[\begin{array}{c}
\sin x_{1} \sin x_{3} \\
b \cos x_{3}+x_{4} \sin x_{1} \cos x_{3}-x_{2} \cos x_{1} \sin x_{3} \\
-\cos x_{3} / \cos x_{1} \\
\left(-x_{4} \sin x_{3}-x_{2} \cos x_{3} \tan x_{1}+b \sin x_{3} \sin x_{1}+2 x_{4} \sin x_{3} \sin ^{2} x_{1}\right) / \cos x_{1}
\end{array}\right] \\
\operatorname{ad}_{f} \mathbf{g}_{2}(\mathbf{x})=\frac{l}{J_{1}}\left[\begin{array}{c}
\cos x_{1} \\
x_{2} \sin x_{1} \\
0 \\
b+2 x_{4} \sin x_{1}
\end{array}\right]
\end{gathered}
$$

Moreover, $\Delta_{0}(\mathbf{x})$ is involutive, since $\left[g_{1}(\mathbf{x}), \mathbf{g}_{2}(\mathbf{x})\right] \in \Delta_{0}(\mathbf{x})$. Hence, since conditions (i), (ii) and (iii) of Theorem 1 are satisfied, the system is feedback-linearizable.

Next, the diffeomorphism $\boldsymbol{\xi}=\left(\xi_{1}, \xi_{2}, \xi_{3}, \xi_{4}\right)^{\top}=\mathscr{C}_{\mathrm{F}}(\mathbf{x})$ that transforms equation (39) to a linear controllable system in Brunovsky form ${ }^{21}$ can be obtained by solving the partial differential equations

$$
\mathrm{L}_{g 1} \lambda_{1}(\mathrm{x})=0, \quad \mathrm{~L}_{g_{2}} \lambda_{2}(\mathrm{x})=0
$$

where $\mathrm{L}_{\mathbf{g}} \mathbf{f}(\mathbf{x}) \triangleq(\partial \mathbf{f}(\mathbf{x}) / \partial \mathbf{x}) \mathbf{g}(\mathbf{x})$ denotes the Lie derivative of $\mathbf{f}(\mathbf{x})$ with respect to $\mathbf{g}(\mathbf{x})$. Since $\Delta_{0}(x)$ is involutive, the Frobenius theorem ${ }^{21}$ guarantees that solutions $\lambda(\mathbf{x})=\left(\lambda_{1}(\mathbf{x}), \lambda_{2}(\mathbf{x})\right)^{\mathrm{T}}$ exist to these equations. One possible solution is

$$
\lambda_{1}(\mathbf{x})=\tan x_{1} \triangleq \xi_{1}, \quad \lambda_{2}(\mathbf{x})=\tan x_{3} \triangleq \xi_{3}
$$

To complete the diffeomorphism, let $\xi_{2} \triangleq \mathrm{L}_{f} \lambda_{1}(\mathbf{x})=x_{2} / \cos ^{2} x_{1}$ and $\xi_{4} \triangleq \mathrm{L}_{f} \lambda_{2}(\mathbf{x})=x_{4} / \cos ^{2} x_{3}$. Hence $\boldsymbol{\xi}=\mathscr{C}_{F}(\mathbf{x})=\left(\tan x_{1}, x_{2} / \cos ^{2} x_{1}, \tan x_{3}, x_{4} / \cos ^{2} x_{3}\right)^{\top}$ and the diffeomorphism $\mathscr{C}_{F}(\mathbf{x})$ : $\mathscr{D} \rightarrow \mathbb{R}^{4}$ is defined globally in $\mathscr{D}$. Then (39) can be transformed to Brunovsky form by defining 
$\mathbf{v}=\left(v_{1}, v_{2}\right)^{\mathrm{T}}$, where $v_{i}=\mathrm{L}_{f}^{2} \lambda_{i}(\mathbf{x})+\sum_{j=1}^{2} \mathrm{~L}_{g} \mathrm{~L}_{f} \lambda_{i}(\mathbf{x}) u_{j}, i=1,2$. A routine calculation shows that

$$
\begin{aligned}
& v_{1}=2 \frac{x_{2}^{2} \sin x_{1}}{\cos ^{3} x_{1}}-x_{4}^{2} \tan x_{1}-\frac{b x_{4}}{\cos x_{1}}+\frac{c}{2} \frac{\sin x_{1}}{\cos ^{2} x_{1}} \cos x_{3}-\frac{l}{J_{1}}\left(u_{1} \frac{\sin x_{1}}{\cos ^{2} x_{1}} \sin x_{3}+u_{2} \frac{1}{\cos x_{1}}\right) \\
& v_{2}=2 x_{4}^{2} \frac{\sin x_{3}}{\cos ^{3} x_{3}}+\frac{1}{\cos x_{1} \cos ^{2} x_{3}}\left(2 x_{2} x_{4} \sin x_{1}+b x_{2}+\frac{c}{2} \sin x_{3}\right)+\frac{l}{J_{1}} \frac{1}{\cos x_{1} \cos x_{3}} u_{1}
\end{aligned}
$$

or

$$
\begin{aligned}
u_{1}= & \frac{J_{1}}{l} \cos x_{1} \cos x_{3}\left[v_{2}-\left(\frac{2 x_{4}^{2} \sin x_{3}}{\cos ^{3} x_{3}}+\frac{2 x_{2} x_{4} \sin x_{1}+b x_{2}+(c / 2) \sin x_{3}}{\cos x_{1} \cos ^{2} x_{3}}\right)\right] \\
u_{2}= & -\frac{J_{1}}{l} \cos x_{1}\left[v_{1}-\left(2 \frac{x_{2}^{2} \sin x_{1}}{\cos ^{3} x_{1}}-x_{4}^{2} \tan x_{1}-\frac{b x_{4}}{\cos x_{1}}+\frac{c}{2} \frac{\sin x_{1}}{\cos ^{2} x_{1}} \cos x_{3}\right)\right] \\
& -\frac{J_{1}}{l} \sin x_{1} \sin x_{3} \cos x_{3}\left[v_{2}-\left(\frac{2 x_{4}^{2} \sin x_{3}}{\cos ^{3} x_{3}}+\frac{2 x_{2} x_{4} \sin x_{1}+b x_{2}+(c / 2) \sin x_{3}}{\cos x_{1} \cos ^{2} x_{3}}\right)\right]
\end{aligned}
$$

Thus utilizing the diffeomorphism $\boldsymbol{\xi}=\mathscr{C}_{\mathrm{F}}(\mathbf{x})$ and substituting (50) and (51) into (39) yields the Brunovsky form

$$
\boldsymbol{\xi}=\left[\begin{array}{llll}
0 & 1 & 0 & 0 \\
0 & 0 & 0 & 0 \\
0 & 0 & 0 & 1 \\
0 & 0 & 0 & 0
\end{array}\right] \boldsymbol{\xi}+\left[\begin{array}{ll}
0 & 0 \\
1 & 0 \\
0 & 0 \\
0 & 1
\end{array}\right]\left[\begin{array}{l}
v_{1} \\
v_{2}
\end{array}\right]
$$

Any linear control law $v$ that stabilizes (52) can be used to stabilize the original system (39) by performing the inverse diffeomorphism $\mathbf{x}=\mathscr{C}_{F}^{-1}(\boldsymbol{\xi})$ along with the control transformation (50), (51). One obvious choice of the linear control law is

$$
\begin{aligned}
& v_{1}=-k_{1} \xi_{1}-k_{2} \xi_{2} \\
& v_{2}=-k_{3} \xi_{3}-k_{4} \xi_{4}
\end{aligned}
$$

with $k_{i}>0, i=1, \ldots, 4$, which yields the control law

$$
\begin{aligned}
u_{1}= & -\frac{J_{1}}{l} \cos x_{1}\left[k_{3} \sin x_{3}+\frac{k_{4} x_{4}}{\cos x_{3}}+\frac{2 x_{4}^{2} \sin x_{3}}{\cos ^{2} x_{3}}+\frac{1}{\cos x_{3}}\left(2 x_{2} x_{4} \sin x_{1}+b x_{2}+\frac{c}{2} \sin x_{3}\right)\right] \\
u_{2}= & \frac{J_{1}}{l}\left(k_{1} \sin x_{1}+k_{2} \frac{x_{2}}{\cos x_{1}}+\frac{2 x_{2}^{3} \sin x_{1}}{\cos ^{2} x_{1}}-x_{4}^{2} \sin x_{1}-b x_{4}+\frac{c}{2} \tan x_{1} \cos x_{3}\right) \\
& +\frac{J_{1}}{l} \sin x_{1} \sin x_{3}\left[k_{3} \sin x_{3}+\frac{k_{4} x_{4}}{\cos x_{3}}+\frac{2 x_{4}^{2} \sin x_{3}}{\cos ^{2} x_{3}}+\frac{1}{\cos x_{3}}\left(2 x_{2} x_{4} \sin x_{1}+b x_{2}+\frac{c}{2} \sin x_{3}\right)\right]
\end{aligned}
$$

in the original system (39). Finally, substituting (55) and (56) into (39) yields 
so that the control law $\mathbf{u}$ in (55), (56) asymptotically stabilizes the fixed-base top to the sleeping motion. Moreover, the stabilization is global on $\mathscr{D}$, since $\mathbf{x}=\mathscr{C}_{\mathrm{F}}^{-1}(\boldsymbol{\xi})$ is a diffeomorphism on $\mathscr{D}$ and the control law $\mathbf{u}$ in (55), (56) is well-defined on $\mathscr{D}$.

\subsection{Hamilton-Jacobi-Bellman theory and zero dynamics}

In order to apply the HJB theory with zero dynamics, we define an artificial output function

$$
\mathbf{y}=\mathbf{h}(\mathbf{x})
$$

where $\mathbf{y} \in \mathbb{R}^{m}$ and $\mathbf{h}(\mathbf{x})=\left(h_{1}(\mathbf{x}), h_{2}(\mathbf{x}), \ldots, h_{m}(\mathbf{x})\right)^{\mathrm{T}}$. For the system (40), (57) consider the performance functional

$$
J\left(\mathbf{x}_{0}, \mathbf{u}(\cdot)\right) \triangleq \int_{0}^{\infty} L(\mathbf{x}(t), \mathbf{u}(t)) \mathrm{d} t
$$

where $x_{0}=\mathbf{x}(0)$ and

$$
L(\mathbf{x}, \mathbf{u}) \triangleq L_{1}(\mathbf{x})+\mathbf{L}_{2}(\mathbf{x}) \mathbf{u}+\mathbf{u}^{\top} \mathbf{R} \mathbf{u}
$$

Here $L_{1}: \mathscr{Q} \rightarrow \mathbb{R}, \mathbf{L}_{2}: \mathscr{X} \rightarrow \mathbb{R}^{1 \times m}$ satisfies $\mathbf{L}_{2}(\mathbf{0})=\mathbf{0}$ and $\mathbf{R} \in \mathbb{R}^{m \times m}$ is a symmetric, positive definite matrix.

We recall the following definitions from Reference 21 . The zero dynamics of the nonlinear system (40), (57) are the dynamics of the system subject to the constraint that the output $\mathbf{y}(t)$ be identically zero, while (40), (57) is said to be minimum phase if its zero dynamics are asymptotically stable. Furthermore, (40), (57) is said to have relative degree $\left\{r_{1}, r_{2}, \ldots, r_{m}\right\}$ at the origin if there exists a neighbourhood $\mathscr{D}_{0}$ of the origin such that, for all $\mathrm{x} \in \mathscr{D}_{0}$,

$$
L_{g i} L_{f}^{k} h_{j}(\mathbf{x})=0, \quad 0<k<r_{j}-1, \quad 1<i, j<m
$$

and the $m \times m$ matrix

$$
\left[\begin{array}{ccc}
L_{g_{1}} L_{f}^{r_{1}-1} h_{1}(\mathbf{x}) & \cdots & L_{g_{m}} L_{f}^{r_{1}-1} h_{1}(\mathbf{x}) \\
\vdots & \vdots & \vdots \\
L_{g_{1}} L_{f}^{r_{m}-1} h_{m}(\mathbf{x}) & \cdots & L_{g_{m}} L_{f}^{r_{m}-1} h_{m}(\mathbf{x})
\end{array}\right]
$$

is non-singular. For the case of relative degree $\left\{r_{1}, r_{2}, \ldots, r_{m}\right\}=\{1,1, \ldots, 1\}$ we define the notation

$$
L_{g} \mathbf{h}(\mathbf{x}) \triangleq\left[\begin{array}{ccc}
L_{g_{1}} h_{1}(\mathbf{x}) & \cdots & L_{g_{m}} h_{1}(\mathbf{x}) \\
\vdots & \vdots & \vdots \\
L_{g_{1}} h_{m}(\mathbf{x}) & \cdots & L_{g_{m}} h_{m}(\mathbf{x})
\end{array}\right]
$$

which in this case is non-singular for all $\mathbf{x} \in \mathscr{D}_{0}$. The following lemma is from Reference 26; see also Reference 23.

\section{Lemma 1}

Assume that the non-linear system (40), (57) is minimum phase with relative degree $\{1,1, \ldots, 1\}$. If the vector field $\mathbf{g}\left(L_{g} \mathbf{h}\right)^{-1}$ is complete, then there exists a diffeomorphism $\mathscr{b}$ : 
$\mathscr{X} \rightarrow \mathscr{R}^{n}, a C^{\infty}$ function $\mathbf{f}_{0}: \mathbb{R}^{n-m} \rightarrow \mathbb{R}^{n-m}$ and $a C^{\infty}$ function $\mathbf{F}: \mathbb{R}^{n-m} \times \mathbb{R}^{m} \rightarrow \mathbb{R}^{(n-m) \times m}$ such that in the co-ordinates

$$
\left[\begin{array}{l}
\mathbf{z} \\
\mathbf{y}
\end{array}\right] \triangleq \mathscr{C}(\mathbf{x})
$$

the differential equation (40) is equivalent to the normal form

$$
\left[\begin{array}{c}
\dot{\mathbf{z}} \\
\dot{\mathbf{y}}
\end{array}\right]=\left[\begin{array}{c}
\mathbf{f}_{0}(\mathbf{z})+\mathbf{F}(\mathbf{z}, \mathbf{y}) \mathbf{y} \\
L_{f} \mathbf{h}(\mathbf{x})
\end{array}\right]+\left[\begin{array}{c}
0 \\
L_{g} \mathbf{h}(\mathbf{x})
\end{array}\right] \mathbf{u}
$$

\section{Theorem $2^{23}$}

Consider the non-linear system defined by equations (40), (57). Assume that the system is minimum phase with relative degree $\{1,1, \ldots, 1\}$ and that the vector field $\mathbf{g}\left(L_{g} \mathbf{h}\right)^{-1}$ is complete. Furthermore, let $\mathbf{P}_{0} \in \mathbb{R}^{m \times m}$ and $\mathbf{R} \in \mathbb{R}^{m \times m}$ be symmetric and positive definite, let $V_{0}(\mathbf{z})$ be a $C^{1}$ positive definite function such that $\left(\partial V_{0}(\mathbf{z}) / \partial \mathbf{z}\right) \mathbf{f}_{0}(\mathbf{z})<0, \mathbf{z} \in \mathbb{R}^{n-m}, \mathbf{z} \neq 0$, and define

$$
\begin{gathered}
\mathbf{L}_{2}^{\mathrm{T}}(\mathbf{x})=\mathbf{R}\left(L_{g} \mathbf{h}\right)^{-1}\left[\mathbf{P}_{0}^{-1} \mathbf{F}^{\mathrm{T}}(\mathbf{z}, \mathbf{y})\left(\frac{\partial V_{0}(\mathbf{z})}{\partial \mathbf{z}}\right)^{\mathrm{T}}+2 L_{f} \mathbf{h}\right] \\
V(\mathbf{x})=V_{0}(\mathbf{z})+\mathbf{y}^{\mathrm{T}} \mathbf{P}_{0} \mathbf{y}
\end{gathered}
$$

where $\mathbf{z}, \mathbf{y}$ and $\mathbf{F}(\mathbf{z}, \mathbf{y})$ are defined in Lemma 1. Then $V(\mathbf{x})$ is a Lyapunov function for the closed-loop system with the control law

$$
\boldsymbol{\phi}(\mathbf{x})=-\frac{1}{2}\left[L_{g} \mathbf{h}(\mathbf{x})\right]^{-1}\left[\mathbf{P}_{0}^{-1} \mathbf{F}^{\mathrm{T}}(\mathbf{z}, \mathbf{y})\left(\frac{\partial V_{0}(\mathbf{z})}{\partial \mathbf{z}}\right)^{\mathrm{T}}+2 L_{f} \mathbf{h}(\mathbf{x})\right]-\mathbf{R}^{-1}\left[L_{g} \mathbf{h}(\mathbf{x})\right]^{\mathrm{T}} \mathbf{P}_{0} \mathbf{h}(\mathbf{x})
$$

which asymptotically stabilizes (40) and minimizes $J\left(\mathbf{x}_{0}, \mathbf{u}(\cdot)\right)$ in the sense that

$$
J\left(\mathbf{x}_{0}, \boldsymbol{\phi}(\mathbf{x}(\cdot))\right)=\min _{\mathbf{u}(\cdot) \in \varphi_{(x}\left(\mathbf{x}_{0}\right)} J\left(\mathbf{x}_{0}, \mathbf{u}(\cdot)\right)=V\left(\mathbf{x}_{0}\right)
$$

for all $\mathbf{x}_{0} \in \mathscr{D}_{0} \subset \mathscr{X}$, where $J\left(\mathbf{x}_{0}, \mathbf{u}(\cdot)\right)$ is defined in (58) and (59) with

$$
L_{1}(\mathbf{x})=\phi^{\mathrm{T}}(\mathbf{x}) \mathbb{R} \boldsymbol{\phi}(\mathbf{x})-\mathrm{L}_{f} V(\mathbf{x}), \quad \mathbf{x} \in \mathscr{X},
$$

and $\mathscr{S}\left(\mathbf{x}_{0}\right)$ is the set of asymptotically stabilizing control laws.

The performance integrand corresponding to the optimal control law (67) is ${ }^{23}$

$$
\begin{aligned}
L(\mathbf{x}, \mathbf{u})= & \left\{\mathbf{u}+\frac{1}{2}\left(L_{g} \mathbf{h}\right)^{-1}\left[\mathbf{P}_{0}^{-1} \mathbf{F}^{\mathrm{T}}(\mathbf{z}, \mathbf{y})\left(\frac{\partial V_{0}(\mathbf{z})}{\partial \mathbf{z}}\right)^{\mathrm{T}}+2 L_{f} \mathbf{h}\right]\right\}^{\mathrm{T}} \mathbf{R} \\
& \times\left\{\mathbf{u}+\frac{1}{2}\left(L_{f} \mathbf{h}\right)^{-1}\left[\mathbf{P}_{0}^{-1} \mathbf{F}^{\mathrm{T}}(\mathbf{z}, \mathbf{y})\left(\frac{\partial V_{0}(\mathbf{z})}{\partial \mathbf{z}}\right)^{\mathrm{T}}+2 L_{f} \mathbf{h}\right]\right\} \\
& -\frac{\partial V_{0}(\mathbf{z})}{\mathbf{z}} \mathbf{f}_{0}(\mathbf{z})+[\mathbf{h}(\mathbf{x})]^{\mathrm{T}} \mathbf{P}_{0}\left(L_{g} \mathbf{h}\right) \mathbf{R}^{-1}\left(L_{g} \mathbf{h}\right)^{\mathrm{T}} \mathbf{P}_{0} \mathbf{h}(\mathbf{x})
\end{aligned}
$$

which is non-negative for all $\mathbf{x}$ and $\mathbf{u}$. Note that $L(\mathbf{x}, \mathbf{u})$ is found a posteriori rather than being specified at the onset. Physical interpretations of $L(\mathbf{x}, \mathbf{u})$ are not known except in simple examples. 
We now apply Theorem 2 to synthesize a control law for (39). Let

$$
\mathbf{y}=\left[\begin{array}{l}
h_{1}(\mathbf{x}) \\
h_{2}(\mathbf{x})
\end{array}\right] \triangleq\left[\begin{array}{l}
k_{2} \tan x_{1}+x_{2} \\
k_{4} \tan x_{3}+x_{4}
\end{array}\right]
$$

where $k_{2}, k_{4}>0$. Defining $\mathbf{z}=\left(z_{1}, z_{2}\right)^{\mathrm{T}} \triangleq\left(\tan x_{1}, \tan \mathbf{x}_{3}\right)^{\mathrm{T}}$, then $\dot{z}=\mathbf{f}_{0}(\mathbf{z})+\mathbf{F}(\mathbf{z}, \mathbf{y}) \mathbf{y}$, where $\mathbf{F}(\mathbf{z}, \mathbf{y})=\mathbf{I}_{2}$ and $\mathbf{f}_{0}(\mathbf{z})=\left(-k_{2} z_{1},-k_{4} z_{2}\right)^{\mathrm{T}}$, with $\mathbf{I}_{2}$ is the $2 \times 2$ identity matrix. It can be shown that the zero dynamics $\dot{z}=\mathbf{f}_{0}(\mathbf{z})$ is asymptotically stable and the corresponding Lyapunov function is

$$
V_{0}(\mathbf{z})=p_{1} z_{1}^{2}+p_{3} z_{2}^{2}
$$

where $p_{1}, p_{3}>0$. Furthermore, the matrix $L_{g} \mathbf{h}(\mathbf{x})$ is non-singular for all $\mathbf{x} \in \mathscr{D}$. Hence equations (39) and (71) form a minimum phase system with relative degree $\{1,1\}$. Then, by letting

$$
\mathbf{P}_{0}=\left[\begin{array}{cc}
p_{2} & 0 \\
0 & p_{4}
\end{array}\right]>0, \quad \mathbf{R}=\left[\begin{array}{cc}
r_{1} & 0 \\
0 & r_{2}
\end{array}\right]>0
$$

the optimal control law $\phi(\mathbf{x})=\left(\phi_{1}(\mathbf{x}), \phi_{2}(\mathbf{x})\right)^{\mathrm{T}}$ in (67) becomes

$$
\begin{aligned}
\phi_{1}(\mathbf{x})= & -\frac{J_{1} \cos x_{1}}{l \cos x_{3}}\left(\frac{p_{3}}{p_{4}} \frac{\sin x_{3}}{\cos ^{3} x_{3}}+k_{4} \frac{x_{4}}{\cos ^{2} x_{3}}+2 x_{2} x_{4} \tan x_{1}+b \frac{x_{2}}{\cos x_{1}}+\frac{c}{2} \frac{\sin x_{3}}{\cos x_{1}}\right) \\
& +\frac{l p_{2}}{J_{1} r_{1}} \sin x_{1} \sin x_{3}\left(k_{2} \tan x_{1}+x_{2}\right)-\frac{l p_{4}}{J_{1} r_{1}} \frac{\cos x_{3}}{\cos x_{1}}\left(k_{4} \tan x_{3}+x_{4}\right) \\
\phi_{2}(\mathbf{x})= & \frac{J_{1}}{l \cos x_{1}}\left(\frac{p_{1}}{p_{2}} \frac{\sin x_{1}}{\cos ^{3} x_{1}}+k_{2} \frac{x_{2}}{\cos ^{2} x_{1}}-\frac{x_{4}^{2}}{2} \sin \left(2 x_{1}\right)-b x_{4} \cos x_{1}+\frac{c}{2} \sin x_{1} \cos x_{3}\right) \\
& +\frac{J_{1}}{l} \sin x_{1} \tan x_{3}\left(\frac{p_{3}}{p_{4}} \frac{\sin x_{3}}{\cos ^{3} x_{3}}+k_{4} \frac{x_{4}}{\cos ^{2} x_{3}}+2 x_{2} x_{4} \tan x_{1}+b \frac{x_{2}}{\cos x_{1}}+\frac{c}{2} \frac{\sin x_{3}}{\cos x_{1}}\right) \\
& +\frac{l p_{2}}{J_{1} r_{2}} \cos x_{1}\left(k_{2} \tan x_{1}+x_{2}\right)
\end{aligned}
$$

A Lyapunov function that guarantees asymptotic stability of the closed-loop system is

$$
V(\mathbf{x})=p_{1} \tan ^{2} x_{1}+p_{3} \tan ^{2} x_{3}+p_{2}\left(k_{2} \tan x_{1}+x_{2}\right)^{2}+p_{4}\left(k_{4} \tan x_{3}+x_{4}\right)^{2}
$$

while the performance integrand (70) is

$$
\begin{aligned}
L(\mathbf{x}, \mathbf{u})= & {\left[\mathbf{u}+\frac{1}{2} \mathbf{R}^{-1} \mathbf{L}_{2}^{\mathrm{T}}(\mathbf{x})\right]^{\mathrm{T}} \mathbf{R}\left[\mathbf{u}+\frac{1}{2} \mathbf{R}^{-1} \mathbf{L}_{2}^{\mathrm{T}}(\mathbf{x})\right]+2 k_{2} p_{1} \frac{\sin ^{2} x_{1}}{\cos ^{4} x_{1}}+2 k_{4} p_{3} \frac{\sin ^{2} x_{3}}{\cos ^{4} x_{3}} } \\
& +\frac{l^{2}}{J_{1}^{2} r_{1}}\left[-p_{2} \sin x_{1} \sin x_{3}\left(k_{2} \tan x_{1}+x_{2}\right)+p_{4} \frac{\cos x_{3}}{\cos x_{1}}\left(k_{4} \tan x_{3}+x_{4}\right)\right]^{2} \\
& +\frac{l^{2}}{J_{1}^{2} r_{2}}\left[p_{2} \cos x_{1}\left(k_{2} \tan x_{1}+x_{2}\right)\right]^{2}
\end{aligned}
$$


where

$$
\mathbf{L}_{2}^{\top}(\mathbf{x})=\left[\begin{array}{l}
2\left(J_{1} / l\right) r_{1} \cos x_{1} / \cos x_{3}\left[\left(p_{3} / p_{4}\right) \sin x_{3} / \cos ^{3} x_{3}+k_{4} x_{4} / \cos ^{2} x_{3}+2 x_{2} x_{4} \tan x_{1}\right. \\
\left.+b x_{2} / \cos x_{1}+(c / 2) \sin x_{3} / \cos x_{1}\right] \\
-2\left(J_{1} / l\right) r_{1} / \cos x_{1}\left[\left(p_{1} / p_{2}\right) \sin x_{1} / \cos ^{3} x_{1}+k_{2} x_{2} / \cos ^{2} x_{1}-\left(x_{4}^{2} / 2\right) \sin \left(2 x_{1}\right)\right. \\
\left.-b x_{4} \cos x_{1}+(c / 2) \sin x_{1} \cos x_{3}\right]-2\left(J_{1} / l\right) r_{2} \sin x_{1} \tan x_{3}\left[k_{4} x_{4} / \cos ^{2} x_{3}\right. \\
\left.+b x_{2} / \cos x_{1}+\left(p_{3} / p_{4}\right) \sin x_{3} / \cos ^{3} x_{3}+2 x_{2} x_{4} \tan x_{1}+(c / 2) \sin x_{3} / \cos x_{1}\right]
\end{array}\right]
$$

Note that the Lyapunov function (76) approaches infinity as $\mathbf{x}$ approaches the boundary of $\mathscr{D}$. This feature is similar to the property of a proper Lyapunov function in $\mathbb{R}^{n}$, and suggests global asymptotic stability for all the initial states in $\mathscr{D}$. Indeed, this observation is evident by taking $\hat{\mathbf{x}}=\left(\tan x_{1}, x_{2}, \tan x_{3}, x_{4}\right)^{\mathrm{T}} \triangleq \mathscr{C}_{Z}(\mathbf{x})$ as transformed state variables. It can be shown that the map $\mathscr{C}_{Z}: \mathscr{D} \rightarrow \mathbb{R}^{4}$ is a global diffeomorphism ${ }^{19}$ from $\mathscr{D}$ onto $\mathbb{R}^{4}$, while its inverse map $\mathscr{C}_{Z}^{-1}$ is a global diffeomorphism from $\mathbb{R}^{4}$ onto $\mathscr{D}$. Hence one can transform the system (39) into the new state variables $\hat{\mathbf{x}}$, and express the control laws (74) and (75) in terms of the new variables $\hat{\mathbf{x}}$. The Lyapunov function (76) in terms of the new variables $\hat{\mathbf{x}}$ is positive definite for all $\hat{\mathbf{x}} \in \mathbb{R}^{4}$ and is proper; in addition, the time derivative of the Lyapunov function is negative definite. Since the control laws (74) and (75) in terms of the new state variables $\hat{\mathbf{x}}$ globally asymptotically stabilize the system for all $\hat{\mathbf{x}} \in \mathbb{R}^{4}$, the closed-loop system (39) with the control laws (74) and (75) is globally asymptotically stable for all $\mathrm{x} \in \mathscr{D}$.

It should be noted that by taking an alternative output function

$$
\mathbf{y}=\left[\begin{array}{l}
k_{2} \tan x_{1}+x_{2} / \cos ^{2} x_{1} \\
k_{4} \tan x_{3}+x_{4} / \cos ^{2} x_{3}
\end{array}\right]
$$

and defining $\mathbf{z}=\left(\tan x_{1}, \tan x_{3}\right)^{\mathrm{T}}$ as before, one can generalize the control laws (55) and (56) obtained from feedback linearization to a larger family as (74) and (75) by using Theorem 2. The Lyapunov function and performance integrand corresponding to the control laws can also be obtained.

Finally, note that the control laws (74) and (75) globally asymptotically stabilize (39) to the sleeping motion irrespective of the values of $b$ and $c$, even if $b^{2}<2 c$, which corresponds to instability of the sleeping motion for the uncontrolled top. As a further special case, global asymptotic stabilization is guaranteed for $b=0$. In this case the top is not spinning at all and is actually an inverted spherical pendulum.

\section{FEEDBACK STABILIZATION WITH ONE FORCE ACTUATOR}

In this section we synthesize a control law that locally asymptotically stabilizes the fixed-base top to the sleeping motion with only one force input. Without loss of generality we assume that the direction of the input force coincides with the $X_{2}$-axis. If the input force is along the $X_{1}$ axis, then one can re-derive the same set of equations using 1-2-3 Euler angles. For a fixedbase top controlled by a horizontal force along the $X_{2}$-direction, the controlled dynamical equations in first-order form are

$$
\dot{x}=\mathbf{f}(\mathbf{x})+\mathbf{g}_{2}(\mathbf{x}) u_{2}
$$

where $\mathbf{f}(\mathbf{x})$ and $\mathbf{g}_{2}(\mathbf{x})$ are as defined previously.

It is straightforward to check that the linearization of (80) about the origin is controllable, which implies that linear control laws derived from the linearization of (80) locally 
asymptotically stabilize $(80)$ to the origin. For the non-linear problem a routine but lengthy calculation shows that $\left[\operatorname{ad}_{f} \mathbf{g}_{2}(\mathbf{x}), \operatorname{ad}_{f}^{2} \mathbf{g}_{2}(\mathbf{x})\right] \notin \Delta_{2}(\mathbf{x}) \triangleq \operatorname{span}\left\{\mathbf{g}_{2}(\mathbf{x}), \operatorname{ad}_{f} \mathbf{g}_{2}(\mathbf{x}), \operatorname{ad}_{f}^{2} \mathbf{g}_{2}(\mathbf{x})\right\}$, which implies that $\Delta_{2}(\mathbf{x})$ is not involutive. Hence $(80)$ is not feedback-linearizable and the feedback linearization method cannot be used for either local or global stabilization.

On the other hand, it is possible to define an artificial scalar output function $y=h(\mathbf{x})$ such that (80) along with this output function forms a system with relative degree one with locally asymptotically stable zero dynamics. A non-linear locally asymptotically stabilizing control law can then be obtained from this approach, and this control law may have a larger domain of attraction compared with the linear control laws.

Alternatively, we apply the Jurdjevic-Quinn (J-Q) technique ${ }^{23,33}$ to obtain locally stabilizing non-linear feedback control laws. For the J-Q technique we state a theorem from Reference 23.

\section{Theorem 3}

Consider the controlled system (40); assume that there exists a $C^{1}$ function $V: \mathscr{X} \rightarrow \mathbb{R}$ and a function $\mathbf{L}_{2}: \mathscr{X} \rightarrow \mathbb{R}^{1 \times m}$ such that

$$
\begin{gathered}
V(\mathbf{0})=0 \\
V(\mathbf{x})>0, \quad \mathbf{x} \in \mathscr{X}, \quad \mathbf{x} \neq \mathbf{0} \\
L_{f_{s}} V(\mathbf{x}) \leqslant \mathbf{0}, \quad \mathbf{x} \in \mathscr{X}, \quad \mathbf{x} \neq \mathbf{0} \\
W \triangleq\left\{\mathbf{x} \in \mathscr{X} \mid L_{f_{s}}^{k+1} V(\mathbf{x})=L_{f_{s}}^{k} L_{g_{i}} V(\mathbf{x})=0, \quad k=0,1, \ldots, i=1, \ldots, m\right\}=\{\mathbf{0}\}
\end{gathered}
$$

where

$$
\mathbf{f}_{s}(\mathbf{x}) \triangleq \mathbf{f}(\mathbf{x})-\frac{1}{2} \mathbf{g}(\mathbf{x}) \mathbf{R}^{-1} \mathbf{L}_{2}^{\mathrm{T}}(\mathbf{x})
$$

Furthermore, define the feedback control law $\mathbf{u}=\boldsymbol{\phi}(\mathbf{x})$, where

$$
\phi(\mathbf{x}) \triangleq-\frac{1}{2} \mathbf{R}^{-1}\left\{\mathbf{L}_{2}^{\mathrm{T}}(\mathbf{x})+\left[L_{g} V(\mathbf{x})\right]^{\mathrm{T}}\right\}
$$

Then there exists a neighbourhood $\mathscr{D}_{0} \subset \mathscr{X}$ of the origin such that for all $\mathbf{x}_{0} \in \mathscr{D}_{0}$, the solution $\mathbf{x}(t)=\mathbf{0}, t \geqslant 0$, of the closed-loop system

$$
\dot{\mathbf{x}}(t)=\mathbf{f}(\mathbf{x}(t))+\mathbf{g}(\mathbf{x}(t)) \phi(\mathbf{x}(t)), \quad \mathbf{x}(0)=\mathbf{x}_{0}, \quad t>0
$$

is asymptotically stable and the performance functional (58) with $L(\mathbf{x}, \mathbf{u})$ defined by (59) and $L_{1}(\mathbf{x})$ defined by $(69)$ is minimized in the sense of $(68)$.

The performance integrand (59) for J-Q-type systems is ${ }^{23}$

$$
L(\mathbf{x}, \mathbf{u})=\left[\mathbf{u}+\frac{1}{2} \mathbf{R}^{-1} \mathbf{L}_{2}^{\mathrm{T}}(\mathbf{x})\right]^{\mathrm{T}} \mathbf{R}\left[\mathbf{u}+\frac{1}{2} \mathbf{R}^{-1} \mathbf{L}_{2}^{\mathrm{T}}(\mathbf{x})\right]-L_{f_{s}} V(\mathbf{x})+\frac{1}{4} L_{g} V(\mathbf{x}) \mathbf{R}^{-1}\left[L_{g} V(\mathbf{x})\right]^{\mathrm{T}}
$$

which is non-negative for all $\mathbf{x} \in \mathbb{R}^{n}$ and $\mathbf{u} \in \mathbb{R}^{m}$.

We use the open-loop Lyapunov function (32) to obtain the J-Q control law and to prove closed-loop stability for the system (80). By assuming $b^{2} \geq 2 c$, we have from Proposition 1 that the sleeping motion of the uncontrolled top is Lyapunov-stable and the Lyapunov function in (32) is positive definite. By letting $\mathbf{L}_{2}(\mathbf{x})=0$ and $\mathbf{R}=r_{2}$, the control law (86) yields

$$
\phi_{2}(x)=\frac{l}{2 J_{1} r_{2}} \cos x_{1}\left(x_{2}+\frac{b}{2} \sin x_{3}\right)
$$

The following proposition examines the largest invariant set in $\mathscr{E}=\{\mathbf{x} \in \mathscr{D}: \dot{V}(\mathbf{x})=0\}$. 


\section{Proposition 2}

Consider the closed-loop system $\dot{x}=f(x)+g_{2}(x) \phi_{2}(x)$, where $f(x)$ and $g_{2}(x)$ are defined by (80) and $\phi_{2}(\mathbf{x})$ is defined by (89). If $V(\mathbf{x})$ is defined by (32), then the largest invariant set in $\varepsilon=\{x \in \mathscr{D}: \dot{V}(x)=0\}$ is $\{0\}$.

Proof. First we note that $\dot{V}(\mathbf{x})=0$ implies $\phi_{2}(\mathbf{x})=0$. Then, from (89) and since cos $x_{1} \neq 0$, $\mathbf{x} \in \mathscr{D}$, it follows that

$$
x_{2}+\frac{b}{2} \sin x_{3}=0
$$

The time derivative of $(90)$ is

$$
x_{4}^{2} \sin x_{1} \cos x_{1}+b x_{4}\left(\cos x_{1}-\frac{1}{2} \cos x_{3}\right)-\frac{c}{2} \sin x_{1} \cos x_{3}=0
$$

From (90) we observe that $x_{2}=0$ if and only if $x_{3}=0$. If $x_{3}(t) \equiv 0$ for some interval of time, then $x_{4}(t)=\dot{x}_{3}(t)=0$. Furthermore, from (91), $x_{4}(t)=0$ implies $x_{1}(t)=0$. Hence, if $x_{2}(t) \equiv 0$ or $x_{3}(t) \equiv 0$, then all the remaining states are identically zero. On the other hand, from (91) we observe that $x_{1}=0$ if and only if $x_{4}=0$. If $x_{1}(t) \equiv 0$ for some interval of time, then $x_{2}(t)=\dot{x}_{1}(t)=0$. Furthermore, from $(90), x_{2}(t)=0$ implies $x_{3}(t)=0$. Hence, if $x_{1}(t) \equiv 0$ or $x_{4}(t) \equiv 0$, then all the remaining states are identically zero. Consequently, we have shown that if $\mathbf{x} \in \mathscr{E}$ and $\mathbf{x} \neq \mathbf{0}$, then none of its components are identically zero.

The time derivative of $(91)$ is

$$
\begin{aligned}
& x_{2}\left[2 x_{4}^{2} \sin ^{2} x_{1}+x_{4} \sin x_{1}\left(\frac{3 b^{2}-3 c}{b}-b \frac{\cos x_{3}}{\cos x_{1}}\right)\right. \\
& \left.\quad+b^{2}-c-\frac{1}{2}\left(b^{2}-c\right) \frac{\cos x_{3}}{\cos x_{1}}-\frac{c}{2} \cos x_{1} \cos x_{3}\right]=0
\end{aligned}
$$

In (92), if $x_{2}=0$, then $x_{1}=x_{3}=x_{4}=0$ as explained earlier. Hence we consider

$$
2 x_{4}^{2} \sin ^{2} x_{1}+x_{4} \sin x_{1}\left(\frac{3 b^{2}-3 c}{b}-b \frac{\cos x_{3}}{\cos x_{1}}\right)+b^{2}-c-\frac{1}{2}\left(b^{2}-c\right) \frac{\cos x_{3}}{\cos x_{1}}-\frac{c}{2} \cos x_{1} \cos x_{3}=0
$$

Eliminating $x_{4}^{2}$ terms in (91) and (93) yields

$$
\frac{3 c-b^{2}}{b} x_{4} \sin x_{1} \cos x_{1}=\left(b^{2}-c\right)\left(\cos x_{1}-\frac{1}{2} \cos x_{3}\right)+c \cos x_{3}\left(\sin ^{2} x_{1}-\frac{1}{2} \cos ^{2} x_{1}\right)
$$

In the special case $b^{2}=3 c$, equation (94) becomes

$$
c \cos x_{1}\left(2-\frac{3}{2} \cos x_{1} \cos x_{3}\right)=0
$$

which results in the contradictory situation $\cos x_{1} \cos x_{3}=4 / 3$. Hence in the special case $b^{2}=3 c$ the largest invariant set in $\mathscr{E}$ is $\{0\}$. If $b^{2} \neq 3 c$, then $x_{4}$ can be expressed as

$$
x_{4}=b \frac{\left(b^{2}-c\right)\left(\cos x_{1}-\frac{1}{2} \cos x_{3}\right)+c \cos x_{3}\left(\sin ^{2} x_{1}-\frac{1}{2} \cos ^{2} x_{1}\right)}{\left(3 c-b^{2}\right) \sin x_{1} \cos x_{1}}
$$

Recall that $\phi_{2}(\mathbf{x})=0, \mathbf{x} \in \mathscr{E}$. Hence the vertical angular momentum is constant, namely

$$
c_{2}=-x_{2} \sin x_{3}+x_{4} \sin x_{1} \cos x_{1} \cos x_{3}+b \cos x_{3} \cos x_{1}
$$


which, by eliminating $x_{2}$ and $x_{4}$ using (90) and (96), yields

$$
-\frac{3 c}{2} \cos ^{2} x_{1} \cos ^{2} x_{3}+2 c \cos x_{1} \cos x_{3}+\frac{1}{2}\left(3 c-b^{2}\right)=c_{4}
$$

where $c_{4}$ is a constant depending on $c_{2}, c$ and $b$. Equation (98) implies

$$
\cos x_{1} \cos x_{3}=c_{5}
$$

where $c_{5}$ is a constant, which further implies that either there is no possible solution to $x_{1}$ and $x_{3}$ (when $\left|c_{5}\right|>1$ ) or the top is under steady precession (when $\left|c_{5}\right| \leqslant 1$ ). If $\left|c_{5}\right|>1$, then the largest invariant set in $\mathscr{E}$ is $\{0\}$. If $\left|c_{5}\right|<1$, then the necessary condition for steady precession given in (16) must be satisfied. Equating (96) and (16) and after some manipulations, we obtain

$$
\begin{aligned}
\cos ^{2} x_{1}\left[\left(b^{2}+c\right) \pm\left(3 c-b^{2}\right)\right. & \sqrt{\left.\left(1-\frac{2 c}{b^{2}} \cos x_{1} \cos x_{3}\right)\right]} \\
= & \left(3 c-b^{2}\right)\left[1 \pm \sqrt{\left(1-\frac{2 c}{b^{2}} \cos x_{1} \cos x_{3}\right)}\right]+\left(b^{2}-3 c\right) \cos x_{1} \cos x_{3}
\end{aligned}
$$

which implies

$$
\cos ^{2} x_{1}=c_{6}
$$

where $c_{6}$ is a constant. Again, if $c_{6}>1$ or $c_{6}<0$, then there is no solution to $x_{1}$, which implies that the largest invariant set in $\mathscr{E}$ is $\{0\}$. If $0 \leqslant c_{6} \leqslant 1$, then $x_{1}$ is a constant; hence we have $x_{2}=\dot{x}_{1}=0$, which contradicts the previous observation that none of the states is zero in $\mathscr{E}$. Thus we have proved that the largest invariant set in $\mathscr{E}$ is $\{0\}$.

Using LaSalle-Krasovskii theorem ${ }^{21}$ and recognizing that the largest invariant set in $\mathscr{E}$ is $\{0\}$, we prove that the control law (89) locally asymptotically stabilizes (80) to the sleeping motion. Note that the control law (89) is locally stabilizing, since the Lyapunov function $V(\mathbf{x})$ does not approach infinity as $\mathbf{x}$ approaches the boundary of $\mathscr{D}$. Hence there may exist initial states in $\mathscr{D}$ such that the control law (89) is not asymptotically stabilizing, which can be checked by simulation results. The performance integrand $(88)$ corresponding to the control law (89) is

$$
L\left(\mathbf{x}, u_{2}\right)=r_{2} u_{2}^{2}+\frac{l^{2}}{4 J_{1}^{2} r_{2}} \cos ^{2} x_{1}\left(x_{2}+\frac{b}{2} \sin x_{3}\right)^{2}
$$

which is non-negative.

\section{SPINNING TOP ON A MOVING CART}

We now consider the case of a spinning top on a moving cart. Suppose that the spinning top is mounted on a moving cart which has mass $M$ and assume that there are two control forces $w_{1}$ and $w_{2}$ applied to the cart along the inertially horizontal directions $X_{1}$ and $X_{2}$ directions (see Figure 2). We will extend the control laws obtained in Section 5 by exploiting d'Alembert's principle ${ }^{15}$ to derive control laws for $w_{1}$ and $w_{2}$ such that $w_{1}$ and $w_{2}$ asymptotically drive the cart-mounted top to the sleeping motion.

Let $\eta_{1}$ and $\eta_{2}$ be the horizontal co-ordinates from a fixed inertial reference point to the vertex of the top. Omitting the details of the derivation, we can write the controlled dynamical 


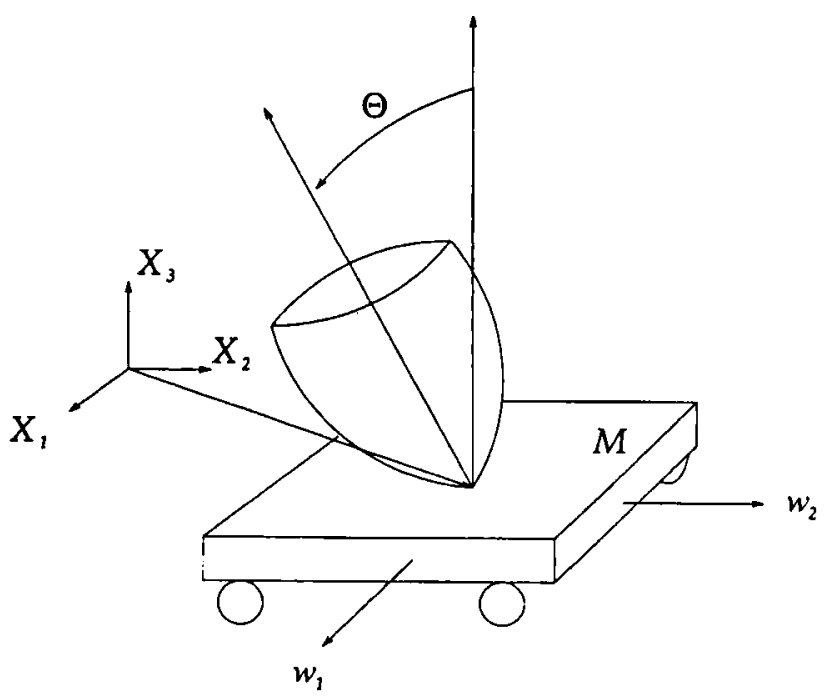

Figure 2. Top mounted on a moving cart

equations for the spinning top on a moving cart using the Routhian method, which yields $(M+m) \ddot{\eta}_{1}+m l\left[-\ddot{\theta} \sin \theta \sin \psi-\left(\dot{\theta}^{2}+\dot{\psi}^{2}\right) \cos \theta \sin \psi\right.$

$$
\begin{aligned}
& +\ddot{\psi} \cos \theta \cos \psi-2 \dot{\theta} \dot{\psi} \sin \theta \cos \psi]=w_{1} \\
& (M+m) \ddot{\eta}_{2}+m l\left(-\ddot{\theta} \cos +\dot{\theta}^{2} \sin \theta\right)=w_{2}
\end{aligned}
$$

$J_{1} \ddot{\theta}+J_{1} \dot{\psi}^{2} \cos \theta \sin \theta+p_{\phi} \dot{\psi} \cos \theta-m g l \sin \theta \cos \psi=l\left(m \ddot{\eta}_{1} \sin \theta \sin \psi+m \ddot{\eta}_{2} \cos \theta\right)$

$J_{1} \ddot{\psi} \cos ^{2} \theta-2 J_{1} \dot{\psi} \dot{\theta} \cos \theta \sin \theta-p_{\phi} \dot{\theta} \cos \theta-m g l \cos \theta \sin \psi=-l m \ddot{\eta}_{1} \cos \theta \cos \psi$

By comparing (105), (106) with (37), (38), one notes that if the inertial forces ${ }^{15} m \ddot{\eta}_{1}$ and $m \ddot{\eta}_{2}$ are taken to be

$$
m \ddot{\eta}_{1}=-\phi_{1}(\mathbf{x}), \quad m \ddot{\eta}_{2}=-\phi_{2}(\mathbf{x})
$$

where $\phi_{1}(\mathbf{x})$ and $\phi_{2}(\mathbf{x})$, defined by (74) and (75) are the globally asymptotically stabilizing control laws that drive the fixed-base top to the sleeping motion, then equations (105) and (106) are globally asymptotically stable for all $\theta$ and $\psi$ less than $90^{\circ}$. Then, according to (103) and (104), the control laws for $w_{1}$ and $w_{2}$ are

$$
\begin{aligned}
w_{1}(\mathbf{x})= & \phi_{1}(\mathbf{x})\left(-\frac{M+m}{m}+\frac{m l^{2}}{J_{1}}\left(1-\cos ^{2} x_{1} \cos ^{2} x_{3}\right)\right)+\phi_{2}(\mathbf{x})\left(\frac{m l^{2}}{J_{1}} \sin x_{1} \cos x_{1} \sin x_{3}\right) \\
& -m l x_{4}^{2} \cos ^{3} x_{1} \sin x_{3}-m l x_{2}^{2} \cos x_{1} \sin x_{3}+m l b x_{2} \cos x_{3} \\
& +m l b x_{4} \sin x_{1} \cos x_{1} \sin x_{3}+\frac{c}{2} m l \cos ^{2} x_{1} \sin x_{3} \cos x_{3} \\
w_{2}(\mathbf{x})= & \phi_{1}(\mathbf{x})\left(\frac{m l^{2}}{J_{1}} \sin x_{1} \cos x_{1} \sin x_{3}\right)+\phi_{2}(\mathbf{x})\left(-\frac{M+m}{m}+\frac{m l^{2}}{J_{1}} \cos ^{2} x_{1}\right)+m l x_{2}^{2} \sin x_{1} \\
& +m l x_{4}^{2} \sin x_{1} \cos ^{2} x_{1}+m l b x_{4} \cos ^{2} x_{1}-\frac{c}{2} m l \sin x_{1} \cos x_{1} \cos x_{3}
\end{aligned}
$$

where $\mathbf{x}=\left(x_{1}, x_{2}, x_{3}, x_{4}\right)^{\top}=(\theta, \dot{\theta}, \psi, \dot{\psi})^{\mathrm{T}}$, and $b$ and $c$ are defined as in Section 2 . 
A straightforward examination shows that by substituting the control laws $w_{1}(\mathbf{x})$ and $w_{2}(\mathbf{x})$ into equations (103) and (104), one obtains (107). Then substituting (107) into equations (105) and (106) yields (37) and (38) with $u_{1}=\phi_{1}(\mathbf{x})$ and $u_{2}=\phi_{2}(\mathbf{x})$, which is globally asymptotically stable for all $\mathbf{x} \in \mathscr{D}$. Hence we conclude that the control laws (108) and (109), applied to the moving cart, drive the cart-mounted top to the sleeping motion.

\section{SIMULATION RESULTS}

In this section we apply the control laws obtained in the previous sections to asymptotically stabilize a spinning top mounted on a moving cart or on a fixed base.

Consider a top with $J_{1}=1 \mathrm{~kg} \mathrm{~m}^{2}, l=1 \mathrm{~m}, m g=3 \mathrm{~N}$ and with total spin $\Omega$ satisfying $J_{3} \Omega=2$ $\mathrm{kg} \mathrm{m} \mathrm{m}^{-1}$. Assume that the top is mounted on a cart with mass $M=0.1 \mathrm{~kg}$ which is held fixed until the control law is applied. Since $b=2 \mathrm{rad} \mathrm{s}^{-1}$ and $c=6 \mathrm{~s}^{-2}$, the top is not spinning sufficiently fast, so that the necessary and sufficient condition $b^{2}>2 c$ for open-loop Lyapunov stability is not satisfied. For simulation we let the initial conditions be $x_{1}(0)=x_{3}(0)=-4 \cdot 58^{\circ}$ and $x_{2}(0)=x_{4}(0)=0 \mathrm{rad} \mathrm{s}^{-1}$, which implies that the slowly spinning top initially has zero transverse angular velocity with $6.48^{\circ}$ of tilt angle $\Theta$. Simulation results show that if the cart is held fixed, the top symmetry axis will hit the surface of the cart at $t \approx 2.3 \mathrm{~s}$. Hence at $t=2.2 \mathrm{~s}$ (when the tilt angle $\Theta$ is about $80^{\circ}$ ) we release the holding forces and apply the feedback control forces to the cart. The control forces are applied according to the control laws (108) and (109) with $\phi_{1}(\mathbf{x})$ and $\phi_{2}(\mathbf{x})$ defined by (74) and (75). The parameters in (74) and (75) are taken to be $k_{2}=k_{4}=1$ $\operatorname{rad~s}^{-1}, p_{1}=p_{3}=1 \mathrm{~N} \mathrm{~m}, p_{2}=p_{4}=1 \mathrm{~kg} \mathrm{~m}^{2}$ and $r_{1}=r_{2}=1 \mathrm{~s} \mathrm{~kg}^{-1}$. Figure 3 shows the tilt angle $\Theta$ and states $x_{1}=\theta$ and $x_{3}=\psi$ with respect to time. Figure 4 shows the corresponding Lyapunov function and Figure 5 shows the holding forces $(t<2.2 \mathrm{~s})$ and the control forces $w_{1}$ and $w_{2}$ $(t>2.2 \mathrm{~s})$. It is seen that the control laws asymptotically drive the cart-mounted top to the sleeping motion as expected. Next we consider a fixed-base top controlled by only one force actuator applied to the centre of mass of the top. With the same values of $J_{1}, l$ and $m g$ as above, we assume $J_{3} \Omega=4 \mathrm{~kg} \mathrm{~m}^{2} \mathrm{~s}^{-1}$ so that $b=4 \mathrm{rad} \mathrm{s}^{-1}$ and $c=6 \mathrm{~s}^{-2}$. Hence $b^{2}>2 c$, which

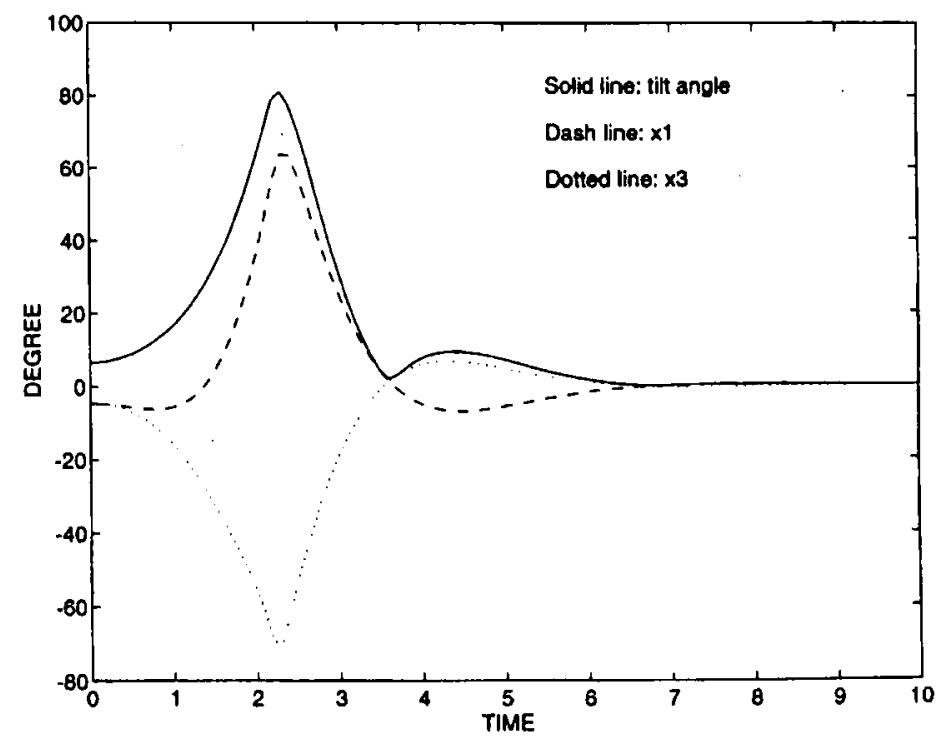

Figure 3. Tilt angle and states $x_{1}$ and $x_{3}$ of a Lagrange top on a moving cart (control forces are applied at $t=2 \cdot 2 \mathrm{~s}$ ) 


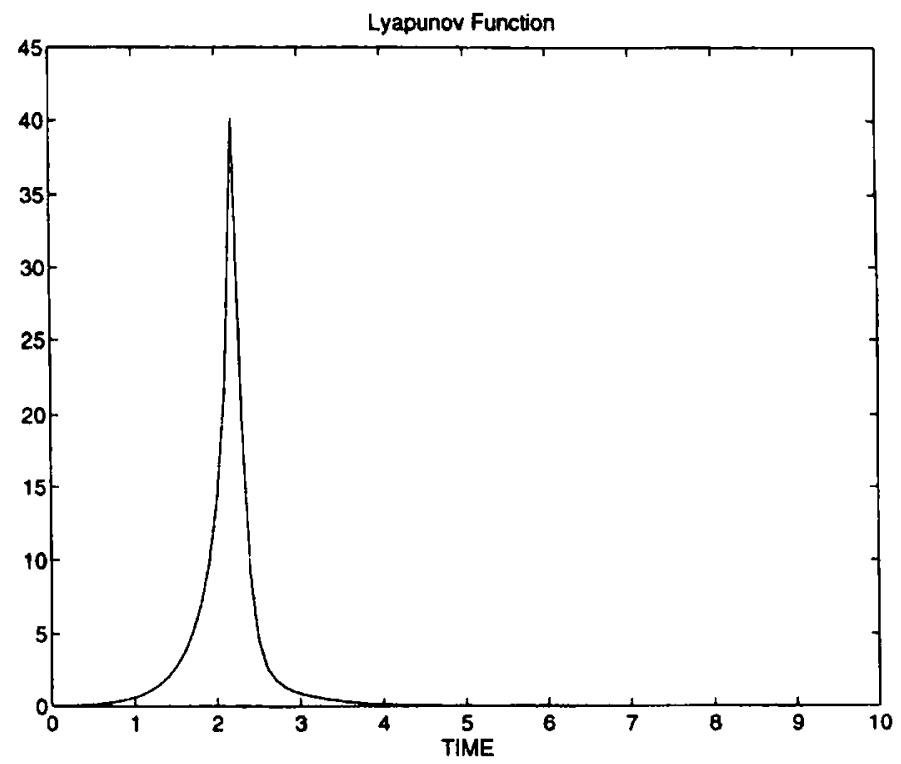

Figure 4. Lyapunov function $V(\mathrm{~N} \mathrm{~m})$ of a Lagrange top on a moving cart (control forces are applied at $t=2.2 \mathrm{~s}$ )

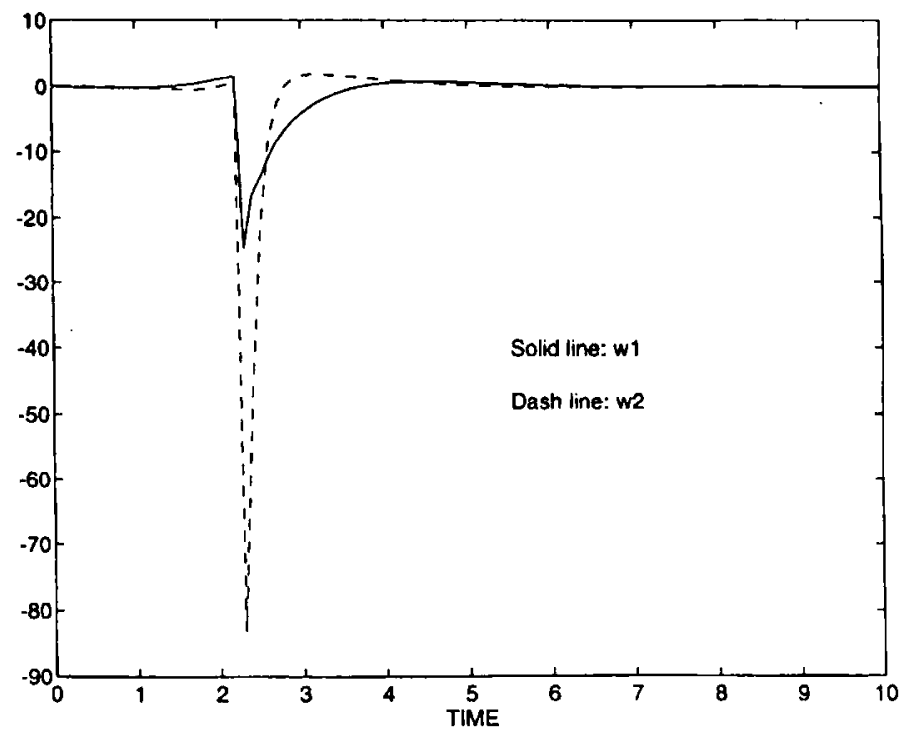

Figure 5. Holding and control forces $w_{1}(\mathrm{~N})$ and $w_{2}(\mathrm{~N})$ of a Lagrange top on a moving cart (control forces are applied at $t=2 \cdot 2 \mathrm{~s})$

implies that the uncontrolled top is Lyapunov stable. Figures 6 and 7 illustrate the case in which the uncontrolled top is initially undergoing steady precession. The control law (89) with $r_{2}=0.5 \mathrm{~s} \mathrm{~kg}^{-1}$ is then applied to the top at $t=15 \mathrm{~s}$. Figure 6 shows the tilt angle $\Theta$ and states $x_{1}=\theta$ and $x_{3}=\psi$ with respect to time. Figure 7 shows the corresponding Lyapunov function and control effort. Figures 6 and 7 show that if the uncontrolled top is Lyapunov stable, then the control law (89) can asymptotically stabilize the top to the sleeping motion even when the tilt 


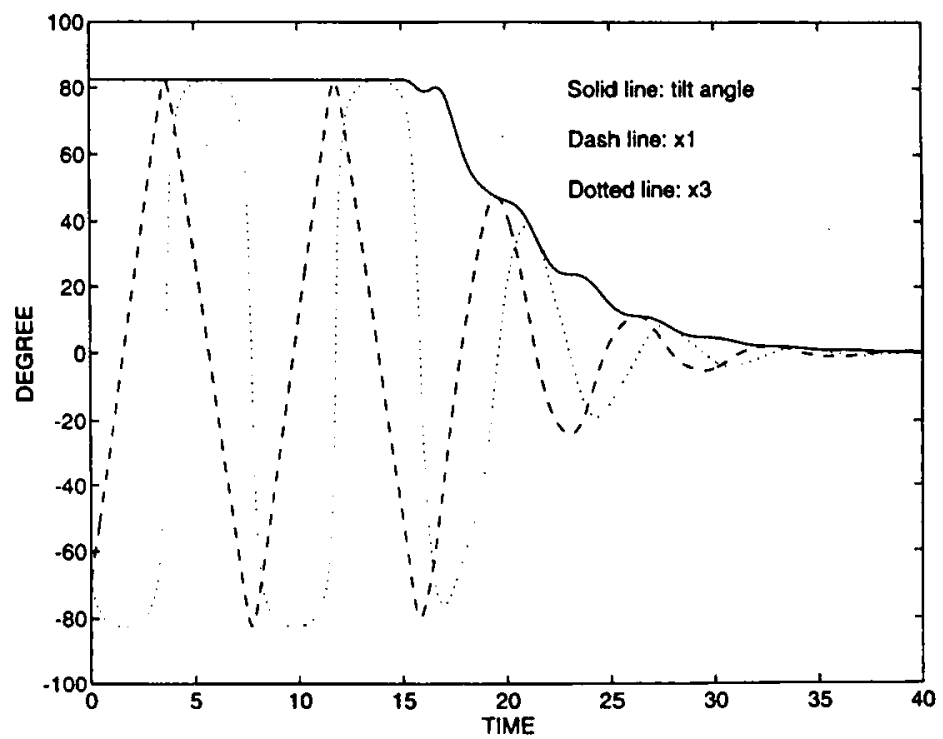

Figure 6. Tilt angles and states $x_{1}$ and $x_{3}$ of a steady precessing fixed-vertex top (control forces are applied at $t=15 \mathrm{~s}$ )

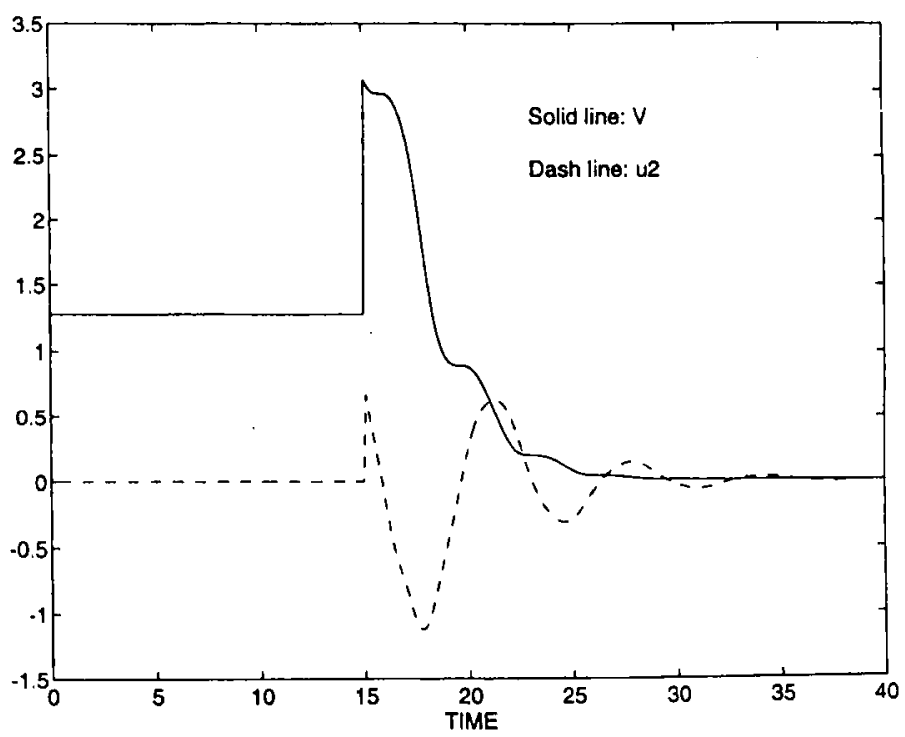

Figure 7. Lyapunov function $V(\mathrm{~N} \mathrm{~m})$ and control $u_{2}(\mathrm{~N})$ of a steady precessing fixed-vertex top (control forces are applied at $t=15 \mathrm{~s}$ )

angle $\Theta$ is close to $90^{\circ}$. Thus the control law (89), although not globally stabilizing in $\mathscr{D}$, has a large domain of attraction. Figures 8 and 9 consider the case in which the top is initially precessing with nutation. The control law (89) with $r_{2}=0.5 \mathrm{~s} \mathrm{~kg}^{-1}$ is applied to the top at $t=15 \mathrm{~s}$. Figure 8 shows the tilt angle $\Theta$ and states $x_{1}=\theta$ and $x_{3}=\psi$ with respect to time. Figure 9 shows the corresponding Lyapunov function and control effort. 


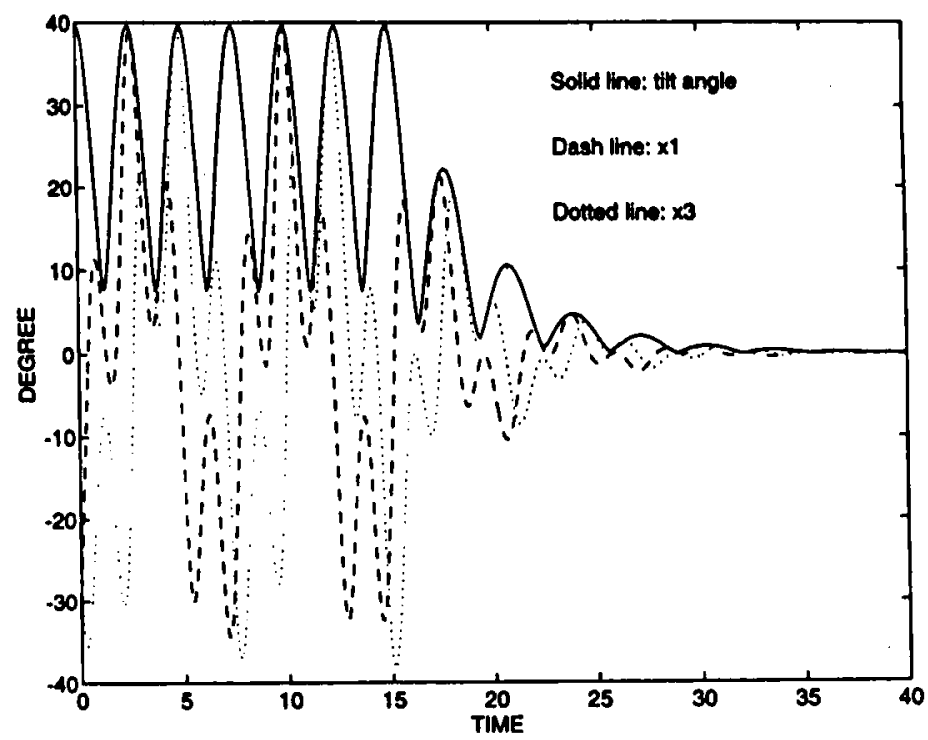

Figure 8. Tilt angle and states $x_{1}$ and $x_{3}$ of a nutating fixed-vertex top (control forces are applied at $t=15 \mathrm{~s}$ )

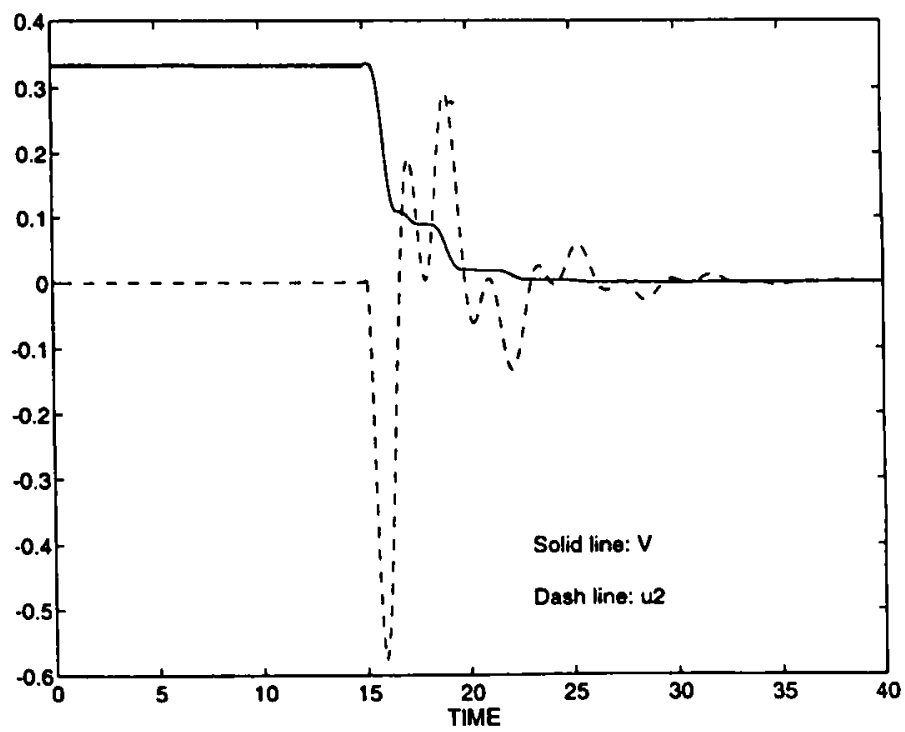

Figure 9. Lyapunov function $V(\mathrm{~N} \mathrm{~m})$ and control $u_{2}(\mathrm{~N})$ of a nutating fixed-vertex top (control forces are applied at $t=15 \mathrm{~s})$

Next we compare the non-linear control law (89) with its linearization at the origin, namely

$$
u_{2}=\frac{l}{2 J_{1} r_{2}}\left(x_{2}+\frac{b}{2} x_{3}\right)
$$

It is straightforward to check that the linearized control law (110) locally asymptotically stabilizes (80). Consider again the open-loop stable top with $b=4 \mathrm{rad} \mathrm{s}^{-1}$ and $c=6 \mathrm{~s}^{-2}$ as in the previous example. Assume that the control is applied at $t=0$ with the initial 
conditions $\mathbf{x}(0)=\left(-0.9 \mathrm{rad}, 0 \mathrm{rad} \mathrm{s}^{-1}, 1.0 \mathrm{rad}, 0 \mathrm{rad} \mathrm{s}^{-1}\right)^{\mathrm{T}}$. Figures 10 and 11 show the tilt angles corresponding to the control laws (89) and (110). It is seen that the control law (89) is stabilizing while the control law (110) is not. Hence Figures 10 and 11 suggest that the $\mathrm{J}-\mathrm{Q}$ control law (89) has a larger domain of attraction than the linearized control law (110).

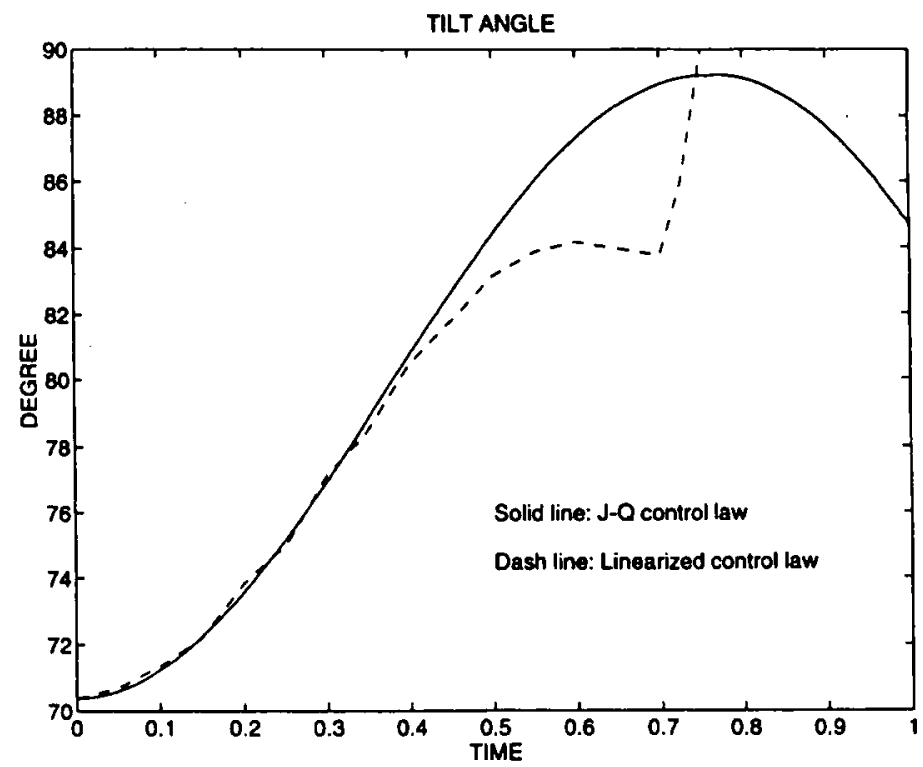

Figure 10. Tilt angle of $\mathrm{J}-\mathrm{Q}$ control and linearized control (from 0 to $1 \mathrm{~s}$ )

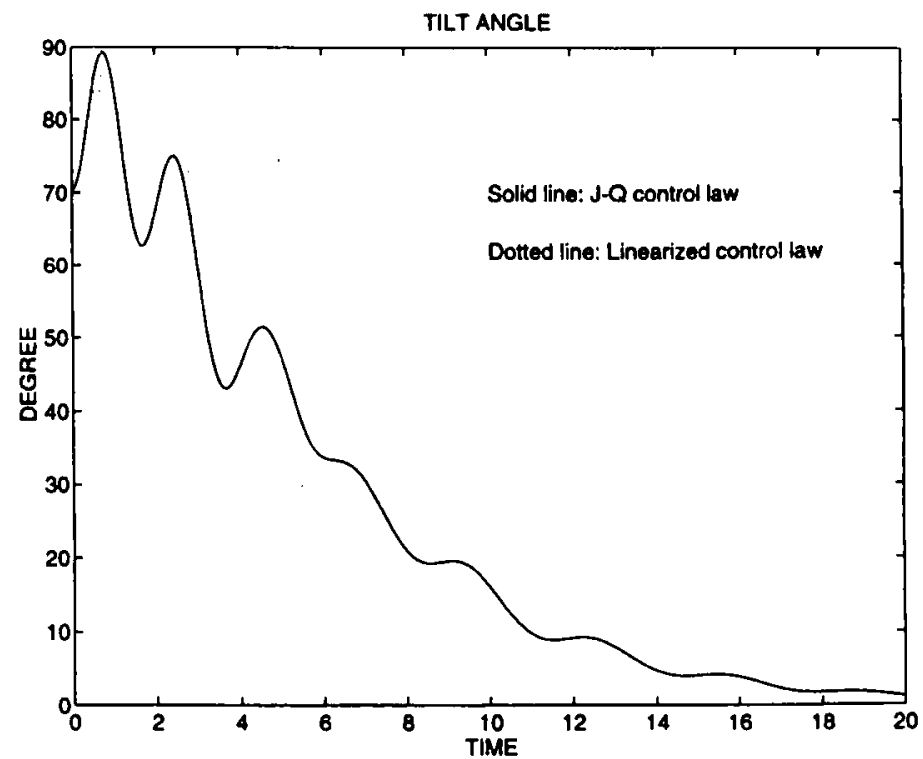

Figure 11. Tilt angle of $\mathrm{J}-\mathrm{Q}$ control and linearized control (from 0 to $20 \mathrm{~s}$ ) 


\section{CONCLUSIONS}

In this paper we derived dynamical equations for Lagrange's top using 2-1-3 Euler angles. For fixed-base tops, necessary and sufficient conditions for Lyapunov stability were given. Control laws that globally asymptotically stabilize the fixed-base top to the sleeping motion using two force inputs applied to the centre of mass of the top were synthesized. Two design strategies, namely feedback linearization and the Hamilton-Jacobi-Bellman theory with zero dynamics, were used. It was shown that the fixed-base top can be asymptotically stabilized to the sleeping motion using two force inputs even if the top is spinning arbitrarily slowly. It was also shown that if the top is spinning sufficiently fast that its free motion is Lyapunov-stable, then it can be locally driven to the sleeping motion by using only one force actuator. The performance functionals optimized by the control laws were also obtained.

For a spinning top mounted on a horizontally moving cart, which is reminiscent of the classical inverted pendulum, we synthesized stabilizing control forces which are applied to the cart and fixed in inertially horizontal directions. A globally asymptotically stabilizing control law was obtained by using d'Alembert's principle to transform the control law obtained for the fixed-base top.

Finally, it should be noted that variations of the spinning top stabilization problem can be formulated by considering alternative actuators. For example, if the spinning top is controlled by body-fixed torques, then the Euler-Poisson formulation with stereographic projection ${ }^{34}$ can be used to synthesize the stabilizing control torques.

\section{ACKNOWLEDGEMENTS}

The authors wish to thank Professor D. T. Greenwood for his helpful discussions. We would also like to thank Mr I. Kolmanovsky for his useful suggestions. This research was supported in part by the Air Force Office of Scientific Research under Grant F49620-92-J-0127.

\section{REFERENCES}

1. Arnold, R. N., and L. Maunder, Gyrodynamics and Its Engineering Applications, Academic, New York, 1961.

2. Crabtree, H., An Elementary Treatment of the Theory of Spinning Tops and Gyroscopic Motion, 2nd edn, Longmans Green, London, 1914.

3. Kettelkamp, L., Spinning Tops, William Morrow, New York, 1968.

4. Klein, F., The Mathematical Theory of the Top, Charles Scribner's Sons, New York, 1897.

5. Macmillan, W. D., Dynamics of Rigid Bodies, McGraw-Hill, New York, 1936.

6. Perry, J., Spinning Tops and Gyroscopic Motion, Dover, New York, 1957.

7. Sethna,P. R., and M. Balachandra, 'Transients in high spin gyroscopic systems', Trans. ASME, J. Appl. Mech. 41, 787-792 (1974).

8. Amold, V. I., Mathematical Methods of Classical Mechanics, 2nd edn, Springer, New York, 1989.

9. Chetayev, N. G., The Stability of Motion, Pergamon, New York, 1961.

10. Ge, Z. M., and Y. J. Wu, "Another theorem for determining the definiteness of sign of functions and its applications to the stability of permanent rotations of a rigid body', Trans. ASME. J. Appl. Mech., 51, 430-434 (1984).

11. Leimanis, E., The General Problem of the Motion of Coupled Rigid Bodies about a Fixed Point, Springer, New York, 1965.

12. Rumiantsev, V. V., 'Stability of permanent rotations of a heavy rigid body', Prikl. Mat. Meh., 20, 51-66 (1956).

13. Ratiu, T., D. D. Holm, J. E. Marsden and A. Weinstein, 'Nonlinear stability of fluid and plasma equilibria', Phys. Rep., 123, 1-116 (1985).

14. Simo, J. C., D. Lewis, T. Ratiu and J. E. Marsden, 'The heavy top; a geometric treatment', Nonlinearity, 5, 1-48 (1992).

15. Greenwood, D. T., Principles of Dynamics, 2nd edn, Prentice-Hall, Englewood Cliffs, NJ, 1988.

16. Synge, J. L., and B. A. Griffith, Principles of Mechanics, 3rd edn, McGraw-Hill, New York, 1959. 
17. Bahar, L. Y., 'Response, stability and conservation laws for the sleeping top problem', J. Sound Vib., 158, 15-34 (1992).

18. Whittaker, E. T., A Treatise on the Analytical Dynamics of Particles and Rigid Bodies, 4th edn, Cambridge University Press, Cambridge, 1964.

19. Tam, T. J., D. C. Cheng and A. Isidori, 'Global extended linearization of nonlinear systems via feedback', IEEE Trans. Automatic Control., AC-30, 808-811 (1985).

20. Su, R., L. R. Hunt and G. Meyer, 'Design for multi-input nonlinear systems', Prog. Math., 27, $268-298$ (1983).

21. Isidori, A., Nonlinear Control Systems, 2nd edn, Springer, New York, 1989.

22. Su, R., 'On the linear equivalents of nonlinear systems', Syst. Control Lett., 2, 48-52 (1982).

23. Wan, C.-J., and D. S. Bernstein, 'Optimal nonlinear feedback control with global stabilization', Dyn. Control, to appear.

24. Hughes, P. C., Spacecraft Attitude Dynamics, Wiley, New York, 1986.

25. Junkins, J. L., and J. D. Tumer, Optimal Spacecraft Rotational Maneuvers, Elsevier, Amsterdam, 1986.

26. Bymes, C. I., and A. Isidori, 'New results and examples in nonlinear feedback stabilization', Syst. Control Lett., 12, 437-442 (1989)

27. Greenwood, D. T., Classical Dynamics, 2nd edn, Prentice-Hall, Englewood Cliffs, NJ, 1988.

28. Hom, R. A., and C. R. Johnson, Matrix Analysis, Cambridge University Press, Cambridge, 1985.

29. Habets, P., N. Rouche and M. Laloy, Stability Theory by Lyapunov's Direct Method, Springer, New York, 1977.

30. Coppola, V. T., C.-J. Wan and D. S. Bernstein, 'A Lyapunov function for the Energy-Casimir method', Proc. Conf. on Decision and Control, IEEE, New York, 1993, pp. 3122-3123.

31. Wan, C.-J., and D. S. Bernstein, 'A family of optimal nonlinear feedback controllers that globally stabilize angular velocity'. Proc. Conf. on Decision and Control, IEEE, New York, 1993, pp. 1143-1148.

32. Wan, C.-J., and D. S. Bernstein, 'Rotational stabilization of a rigid body using two torque actuators', Proc. Conf. on Decision and Control, IEEE, New York, 1993, pp. 3111-3116.

33. Bacciotti, A., Series on Advances in Mathematics for Applied Sciences, Local Stabilizability of Nonlinear Control Systems, Vol. 8, World Scientific, Singapore, 1992.

34. Tsiotras, P., and J. M. Longuski, 'On attitude stabilization of symmetric spacecraft with two control torques', Proc. Am. Control Conf., 1993, pp. 44-50. 